\title{
HATS-50b through HATS-53b: Four Transiting Hot Jupiters Orbiting G-type Stars Discovered by the HATSouth Survey*
}

Th. Henning ${ }^{1}$, L. Mancini ${ }^{1,2,3}$ (1) P. Sarkis ${ }^{1}$, G. Á. Bakos ${ }^{4,18}$ (1) J. D. Hartman ${ }^{4}$ (1) D. Bayliss ${ }^{5}$, J. Bento ${ }^{6}$, W. Bhatti ${ }^{4}$ (10, R. Brahm ${ }^{7,8}$ (1) S. Ciceri ${ }^{9}$, Z. Csubry ${ }^{4}$, M. de Val-Borro ${ }^{4}$ (1) N. Espinoza ${ }^{7,8}$ (1) , B. J. Fulton ${ }^{10}$ (1) , A. W. Howard $^{10}{ }^{(1)}$,

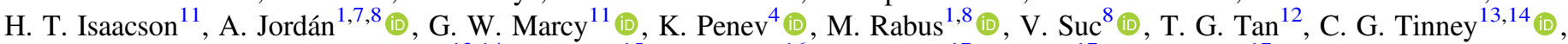
D. J. Wright ${ }^{13,14}$, G. Zhou ${ }^{15}$, S. Durkan ${ }^{16}$ (D), J. Lazar ${ }^{17}$, I. Papp ${ }^{17}$, and P. Sari ${ }^{17}$

${ }^{1}$ Max Planck Institute for Astronomy, Königstuhl 17, D-69117-Heidelberg, Germany; henning@mpia.de

${ }^{2}$ Department of Physics, University of Rome Tor Vergata, Via della Ricerca Scientifica 1, I-00133-Rome, Italy

${ }^{3}$ INAF-Astrophysical Observatory of Turin, via Osservatorio 20, I-10025-Pino Torinese, Italy

${ }^{4}$ Department of Astrophysical Sciences, Princeton University, NJ 08544, USA

${ }^{5}$ Department of Physics, University of Warwick, Coventry CV4 7AL, UK

${ }^{6}$ Research School of Astronomy and Astrophysics, Australian National University, Canberra, ACT 2611, Australia

7 Millennium Institute of Astrophysics, Av. Vicuña Mackenna 4860, 7820436 Macul, Santiago, Chile

${ }^{8}$ Instituto de Astrofísica, Pontificia Universidad Católica de Chile, Av. Vicuña Mackenna 4860, 7820436 Macul, Santiago, Chile

${ }^{9}$ Department of Astronomy, Stockholm University, SE-106 91 Stockholm, Sweden

${ }^{10}$ California Institute of Technology, Pasadena, CA 91125, USA

${ }^{11}$ Astronomy Department, University of California, Berkeley, CA, 94720, USA

${ }^{12}$ Perth Exoplanet Survey Telescope, Perth, Australia

${ }_{13}^{13}$ Australian Centre for Astrobiology, School of Physics, University of New South Wales, NSW 2052, Australia

${ }^{14}$ Exoplanetary Science at UNSW, School of Physics, University of New South Wales, NSW 2052, Australia

${ }_{16}^{5}$ Harvard-Smithsonian Center for Astrophysics, 60 Garden Street, Cambridge, MA 02138, USA

${ }^{16}$ Astrophysics Research Centre, Queens University, Belfast, Belfast, Northern Ireland, UK

${ }^{17}$ Hungarian Astronomical Association, 1451 Budapest, Hungary

Received 2017 November 21; revised 2017 December 11; accepted 2017 December 11; published 2018 January 23

\begin{abstract}
We report the discovery of four close-in transiting exoplanets (HATS-50b through HATS-53b), discovered using the HATSouth three-continent network of homogeneous and automated telescopes. These new exoplanets belong to the class of hot Jupiters and orbit G-type dwarf stars, with brightness in the range $V=12.5-14.0 \mathrm{mag}$. While HATS-53 has many physical characteristics similar to the Sun, the other three stars appear to be metal-rich $([\mathrm{Fe} / \mathrm{H}]=0.2-0.3)$, larger, and more massive. Three of the new exoplanets, namely HATS-50b, HATS-51b, and HATS-53b, have low density (HATS-50b: $0.39 \pm 0.10 M_{\mathrm{J}}, 1.130 \pm 0.075 R_{\mathrm{J}}$; HATS-51b: $0.768 \pm 0.045 M_{\mathrm{J}}$, $1.41 \pm 0.19 R_{\mathrm{J}}$; HATS-53b: $0.595 \pm 0.089 M_{\mathrm{J}}, 1.340 \pm 0.056 R_{\mathrm{J}}$ ) and similar orbital periods $(3.8297$ days, 3.3489 days, 3.8538 days, respectively). Instead, HATS-52b is more dense (mass $2.24 \pm 0.15 M_{\mathrm{J}}$ and radius $\left.1.382 \pm 0.086 R_{\mathrm{J}}\right)$ and has a shorter orbital period (1.3667 days). It also receives an intensive radiation from its parent star and, consequently, presents a high equilibrium temperature $\left(T_{\mathrm{eq}}=1834 \pm 73 \mathrm{~K}\right)$. HATS-50 shows a marginal additional transit feature consistent with an ultra-short-period hot super Neptune (upper mass limit $0.16 M_{\mathrm{J}}$ ), which will be able to be confirmed with TESS photometry.
\end{abstract}

Key words: stars: individual (HATS-50, HATS-51, HATS-52, HATS-53) - techniques: photometric

Supporting material: machine-readable tables

\section{Introduction}

Ground-based transit surveys, based on small robotic telescopes, are a versatile tool for the detection of transiting exoplanets and the precise measurement of planetary radii and

\footnotetext{
* The HATSouth network is operated by a collaboration consisting of Princeton University (PU), the Max Planck Institute für Astronomie (MPIA), the Australian National University (ANU), and the Pontificia Universidad Católica de Chile (PUC). The station at Las Campanas Observatory (LCO) of the Carnegie Institute is operated by PU in conjunction with PUC, the station at the High Energy Spectroscopic Survey (H.E.S.S.) site is operated in conjunction with MPIA, and the station at Siding Spring Observatory (SSO) is operated jointly with ANU. Based in part on observations made with the ESO $3.6 \mathrm{~m}$, the NTT, the MPG $2.2 \mathrm{~m}$ and Euler $1.2 \mathrm{~m}$ Telescopes at the ESO Observatory in La Silla. Based in part on observations made with the $3.9 \mathrm{~m}$ Anglo-Australian Telescope and the ANU $2.3 \mathrm{~m}$ Telescope, both at SSO. Based in part on observations made with the Keck I Telescope at Mauna Kea Observatory in Hawaii. Based in part on observations obtained with the facilities of the Las Cumbres Observatory Global Telescope and with the Perth Exoplanet Survey Telescope.

${ }^{18}$ Packard Fellow.
}

masses. They have provided key contributions to exoplanetary science by discovering extremely interesting objects (e.g., WASP-12b: Hebb et al. 2009; GJ 1124b: Charbonneau et al. 2009, HAT-P-11b: Bakos et al. 2010), and are still revealing astonishing planetary systems (some of the most recent ones are, for example, GJ 1132: Berta-Thompson et al. 2015; XO-2: Burke et al. 2007; Desidera et al. 2014; Damasso et al. 2015; WASP-47: Hellier et al. 2012; Becker et al. 2015; Trappist-1: Gillon et al. 2016; KELT-9: Gaudi et al. 2017).

Due to observational and instrumental limitations, these surveys are particularly sensitive for detecting hot Jupiters, which are a class of exoplanets formed by gas giant planets, similar to Jupiter in terms of size, mass, and composition, but having shorter orbital periods ( $P_{\text {orb }}<10$ days). Considering the proximity to their parent stars and because they are more massive and larger than ice and rocky planets, hot Jupiters are often excellent targets for the follow-up characterization of their physical properties and atmospheres. 
Table 1

Summary of Photometric Observations

\begin{tabular}{|c|c|c|c|c|c|}
\hline Instrument/Field ${ }^{\mathrm{a}}$ & Date(s) & \# Images & $\begin{array}{c}\text { Cadence }^{\mathrm{b}} \\
\text { (s) }\end{array}$ & Filter & $\begin{array}{c}\text { Precision }^{\mathrm{c}} \\
(\mathrm{mmag})\end{array}$ \\
\hline \multicolumn{6}{|l|}{ HATS-50 } \\
\hline HS-2.4/G580 & 2010 Mar-2011 Aug & 6072 & 294 & $r$ & 12.6 \\
\hline HS-6.4/G580 & 2010 Mar-2011 May & 742 & 297 & $r$ & 14.1 \\
\hline HS-1.3/G625 & 2012 Jun-2012 Oct & 4662 & 291 & $r$ & 13.2 \\
\hline HS-3.3/G625 & 2012 Jun-2012 Oct & 5357 & 293 & $r$ & 13.0 \\
\hline LCOGT $1 \mathrm{~m}+\mathrm{CTIO} /$ sinistro & 2015 May 11 & 57 & 226 & $i$ & 1.3 \\
\hline LCOGT $1 \mathrm{~m}+\mathrm{SAAO} / \mathrm{SBIG}$ & 2015 Jun 06 & 136 & 150 & $i$ & 4.1 \\
\hline \multicolumn{6}{|l|}{ HATS-51 } \\
\hline HS-1.2/G601 & 2011 Aug-2012 Jan & 4806 & 296 & $r$ & 6.2 \\
\hline HS-3.2/G601 & 2011 Aug-2012 Jan & 4062 & 296 & $r$ & 6.6 \\
\hline HS-5.2/G601 & 2011 Aug-2012 Jan & 3083 & 290 & $r$ & 6.8 \\
\hline \multicolumn{6}{|l|}{ HATS-52 } \\
\hline HS-2.1/G606 & 2012 Feb-2012 Jun & 3753 & 291 & $r$ & 9.1 \\
\hline HS-4.1/G606 & 2012 Feb-2012 Jun & 2778 & 300 & $r$ & 11.8 \\
\hline HS-6.1/G606 & 2012 Feb-2012 Jun & 1184 & 299 & $r$ & 9.8 \\
\hline PEST $0.3 \mathrm{~m}$ & $2015 \mathrm{Feb} 06$ & 193 & 132 & $R_{C}$ & 12.8 \\
\hline LCOGT $1 \mathrm{~m}+\mathrm{CTIO} /$ sinistro & 2015 May 12 & 38 & 226 & $i$ & 4.1 \\
\hline LCOGT $1 \mathrm{~m}+\mathrm{SSO} / \mathrm{SBIG}$ & 2015 May 13 & 53 & 195 & $i$ & 1.4 \\
\hline LCOGT $1 \mathrm{~m}+\mathrm{CTIO} /$ sinistro & 2015 May 16 & 42 & 226 & $i$ & 1.9 \\
\hline LCOGT $1 \mathrm{~m}+\mathrm{CTIO} /$ sinistro & 2015 Oct 23 & 100 & 54 & $i$ & 6.4 \\
\hline \multicolumn{6}{|l|}{ HATS-53 } \\
\hline HS-2.4/G610 & 2011 Apr-2013 Jul & 5496 & 280 & $r$ & 10.8 \\
\hline HS-4.4/G610 & 2013 Jan-2013 Jul & 3739 & 323 & $r$ & 10.8 \\
\hline
\end{tabular}

Notes.

${ }^{\text {a }}$ For HATSouth data, we list the HATSouth unit, CCD, and field name from which the observations are taken. HS-1 and -2 are located at Las Campanas Observatory in Chile, HS-3 and -4 are located at the H.E.S.S. site in Namibia, and HS-5 and -6 are located at Siding Spring Observatory in Australia. Each unit has four CCDs. Each field corresponds to one of 838 fixed pointings used to cover the full $4 \pi$ celestial sphere. All data from a given HATSouth field and CCD number are reduced together, while detrending through External Parameter Decorrelation (EPD) is done independently for each unique unit $+\mathrm{CCD}+$ field combination.

$\mathrm{b}$ The median time between consecutive images rounded to the nearest second. Due to factors such as weather, the day-night cycle, guiding, and focus corrections, the cadence is only approximately uniform over short timescales.

${ }^{c}$ The rms of the residuals from the best-fit model.

Thanks to the efforts of various teams (e.g., HATNet: Bakos et al. 2004; WASP: Pollacco et al. 2006; KELT: Pepper et al. 2007, 2012; MEarth: Charbonneau et al. 2009; QES: Alsubai et al. 2013; NGTS: Wheatley et al. 2017) who set up and ran ground-based surveys for many years, we currently know of roughly 300 hot Jupiters, whose physical and orbital parameters have been well determined. However, they represent less than $10 \%$ of $\approx 3500$ confirmed exoplanets. ${ }^{19}$ In fact, one of the greatest achievements obtained by the Kepler space-telescope survey (Borucki et al. 2011) was to establish the statistical abundance of the different classes of exoplanets in the Galaxy, revealing that giant planets are rarer than small-size rocky and Neptunian-type planets (Dressing \& Charbonneau 2013; Fressin et al. 2013). However, even though hot Jupiters are relatively

\footnotetext{
${ }^{19}$ Data taken from the NASA Exoplanet Archive: https://exoplanetarchive. ipac.caltech.edu/.
}

rare, there are many open questions that make these bodies extremely interesting to study.

Debated are the theories that have been proposed to explain their formation and evolution, including in situ scenarios (Bodenheimer et al. 2000; Batygin et al. 2016; Boley et al. 2016) and physical mechanisms that reasonably forced them to migrate, from the snowline, so close to their parent star (Lin et al. 1996; Rasio \& Ford 1996; Fabrycky \& Tremaine 2007; Chatterjee et al. 2008; Marzari \& Nelson 2009; Bitsch \& Kley 2011). It remains to be fully understood why giant exoplanets with similar masses present such a wide range of radii (see Thorngren \& Fortney 2017). Particularly intriguing is, finally, the fact that the most recent studies of hot Jupiters' atmospheres have shown a wide range of different results, including Rayleigh scattering, $\mathrm{Na}$ and $\mathrm{K}$ absorption, detection of molecules, like $\mathrm{H}_{2} \mathrm{O}$ and titanium oxide, and flat 

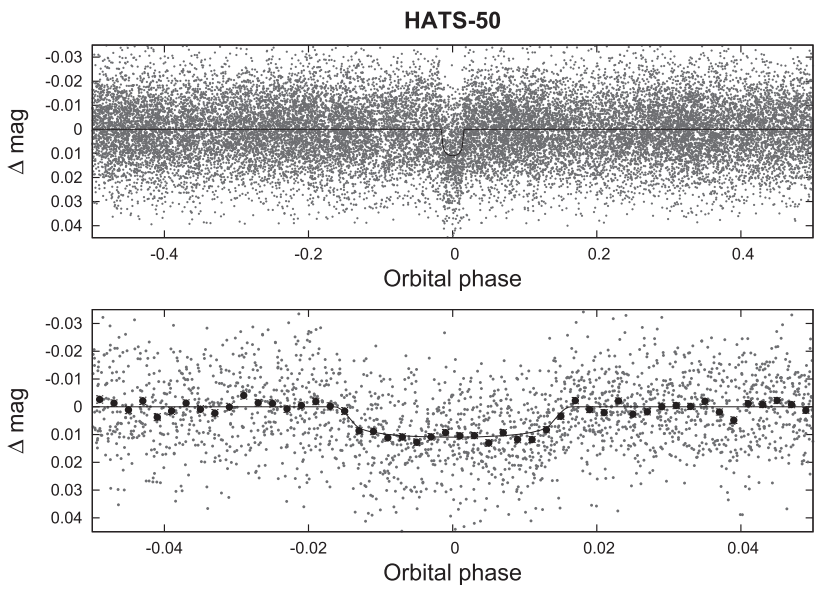

HATS-52
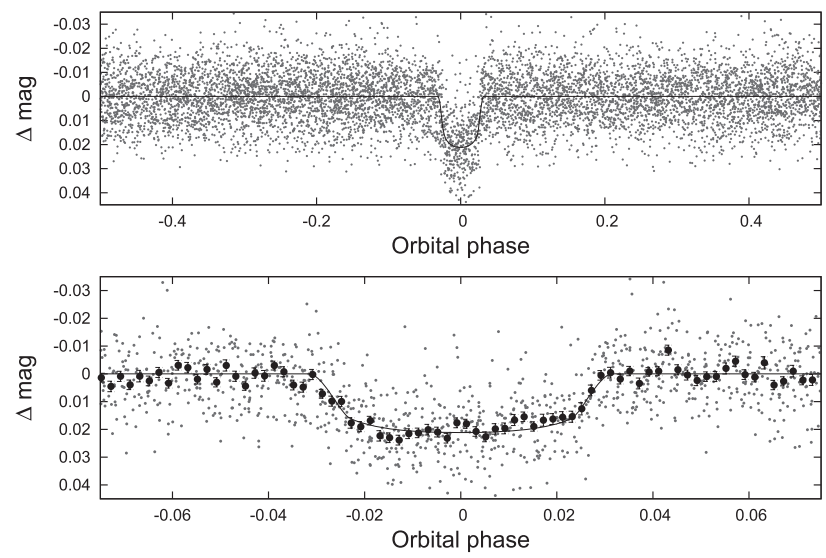
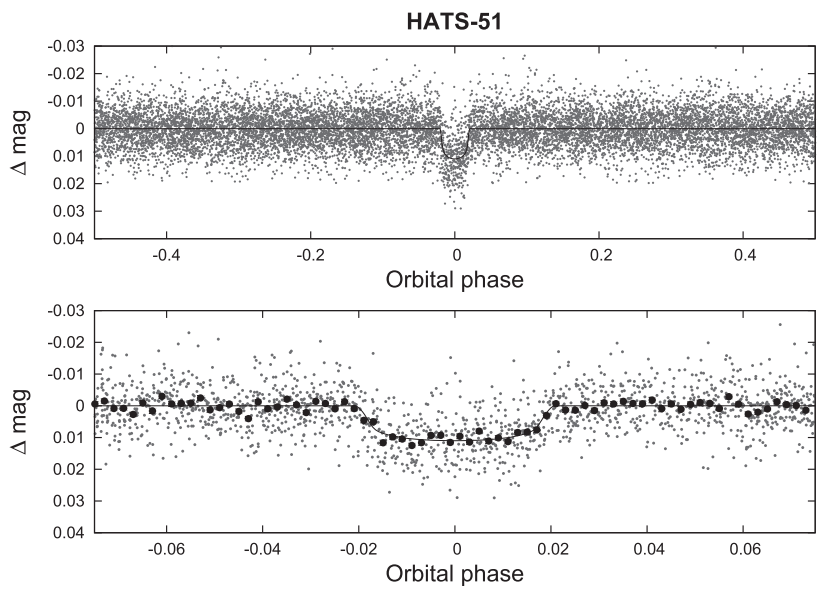

HATS-53
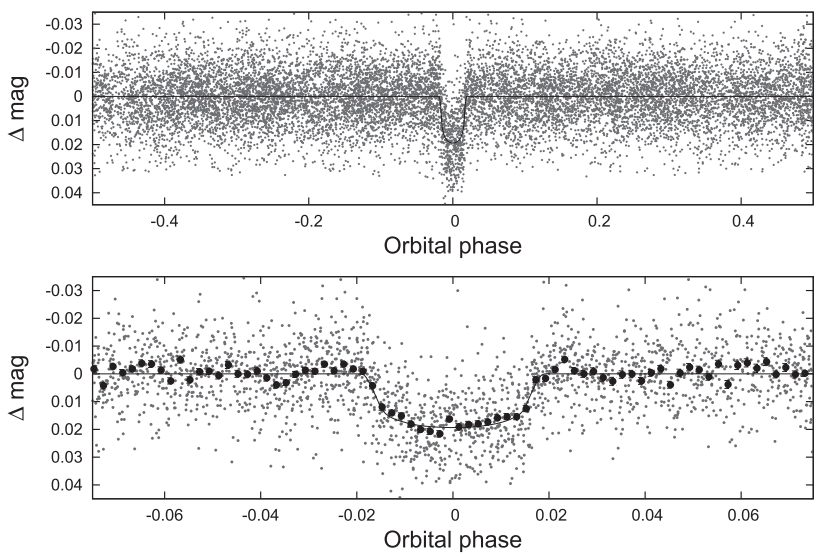

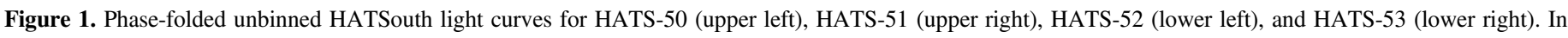

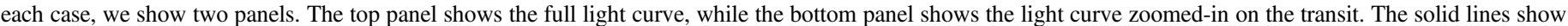
the model fits to the light curves. The dark filled circles in the bottom panels show the light curves binned in phase with a bin size of 0.002 .

transmission spectra probably caused by the presence of thick clouds or hazes (Sing et al. 2016; Sedaghati et al. 2017).

In order to give the right answers to these and other theoretical and phenomenological questions concerning hot Jupiters, it is mandatory to have a large enough sample for statistical studies. Ground-based surveys have been conceived for this purpose and the current challenge is to try to fill all the parameter space of exoplanet properties, in particular those zones where the investigation is particularly hard due to observational biases.

In this context, we are undertaking the HATSouth project with the aim to detect new transiting exoplanet systems. The HATSouth survey consists of a network of 24 homogeneous telescopes, which are mounted on six automated units distributed in pairs over three continents (South America, Africa, and Australia). The large number of telescopes and the wide separation between the HATSouth stations increases the sensitivity to exoplanets orbiting faint stars (12 mag $<V<16 \mathrm{mag}$ ) and having long orbital periods ( $>10$ days) (Bakos et al. 2013).

In this work, we present four new transiting extrasolar planets: HATS-50b, HATS-51b, HATS-52b, and HATS-53b. The paper is organized as follows: In Section 2, we describe the detection of the photometric transit signal by the HATSouth survey and the spectroscopic and photometric follow-up observations performed to confirm the exoplanetary nature of the candidates. Then, in Section 3, we jointly analyze the data to determine the stellar and planetary parameters, ruling out false positive scenarios. Our results are finally summarized and discussed in Section 4.

\section{Observations}

\subsection{The HATSouth Survey}

The four new exoplanets reported in this work have been detected thanks to the HATSouth survey ${ }^{20}$ (Bakos et al. 2013). This is a network of robotic wide-field telescopes, composed of six identical units located in three stations. The stations are distributed over three continents in the southern hemisphere, i.e., Las Campanas Observatory (LCO) in Chile, the H.E.S.S. site in Namibia, and Siding Spring Observatory (SSO) in Australia. Each unit consists of a single mount with four $18 \mathrm{~cm}$ Takahashi astrographs with a focal length of $500 \mathrm{~mm}$, and four Apogee U16M Alta CCD cameras, which have $4 \mathrm{k} \times 4 \mathrm{k}$ pixels of size $9.0 \mu \mathrm{m}$. With a plate scale of $3.7 \operatorname{arcsec}_{\text {pixel }}{ }^{-1}$, the total mosaic field-of-view on the sky is $8^{\circ} \times 8^{\circ}$. The survey operates in the visual, through Sloan- $r$ filters, and the scientific images are obtained using an exposure time of 4 minutes. They are then automatically calibrated with bias, dark and flat images and are stored in the HATSouth archive at Princeton University. Each stellar field is monitored for roughly 2-3 months from each station, in order to get a $24 \mathrm{hr}$ coverage, thus exploiting the great advantage coming from their large separation in longitude. Once a

\footnotetext{
${ }^{20}$ http://hatsouth.org/
} 
Table 2

Summary of Spectroscopy Observations

\begin{tabular}{|c|c|c|c|c|c|c|}
\hline Instrument & UT Date(s) & \# Spec. & $\begin{array}{l}\text { Res. } \\
\Delta \lambda / \lambda / 1000\end{array}$ & $\mathrm{~S} / \mathrm{N}$ Range $^{\mathrm{a}}$ & $\begin{array}{c}\gamma_{\mathrm{RV}}{ }^{\mathrm{b}} \\
\left(\mathrm{km} \mathrm{s}^{-1}\right)\end{array}$ & $\begin{array}{l}\text { RV Precision } \\
\quad\left(\mathrm{m} \mathrm{s}^{-1}\right)\end{array}$ \\
\hline \multicolumn{7}{|l|}{ HATS-50 } \\
\hline ANU $2.3 \mathrm{~m} / \mathrm{WiFeS}$ & 2014 Jun $3-5$ & 3 & 7 & $21-90$ & -23.4 & 4000 \\
\hline ANU $2.3 \mathrm{~m} / \mathrm{WiFeS}$ & 2014 Jun 4 & 1 & 3 & 76 & $\ldots$ & $\ldots$ \\
\hline Euler $1.2 \mathrm{~m} / \mathrm{CORALIE}$ & 2014 Jun-Sep & $4^{\mathrm{d}}$ & 60 & $7-13$ & -20.176 & 73 \\
\hline MPG $2.2 \mathrm{~m} /$ FEROS & 2014 Jul-2016 Sep & $32^{\mathrm{d}}$ & 48 & $14-55$ & -20.250 & 72 \\
\hline Keck I $10 \mathrm{~m} / \mathrm{HIRES}+\mathrm{I}_{2}$ & 2014 Sep-2015 Jul & 7 & 48 & $110-155$ & $\ldots$ & 29 \\
\hline Keck I 10 m/HIRES & $2015 \mathrm{Jul} 5$ & 1 & 48 & 70 & $\cdots$ & $\cdots$ \\
\hline \multicolumn{7}{|l|}{ HATS-51 } \\
\hline ANU $2.3 \mathrm{~m} / \mathrm{WiFeS}$ & 2014 Oct 7 & 1 & 3 & 46 & $\cdots$ & $\ldots$ \\
\hline ANU $2.3 \mathrm{~m} / \mathrm{WiFeS}$ & 2014 Oct $8-12$ & 4 & 7 & $33-67$ & 2.0 & 4000 \\
\hline Euler $1.2 \mathrm{~m} / \mathrm{CORALIE}$ & 2014 Oct-2016 Nov & $24^{\mathrm{d}}$ & 60 & $8-30$ & 3.086 & 55 \\
\hline MPG $2.2 \mathrm{~m} /$ FEROS & 2014 Dec-2015 Feb & 14 & 48 & $60-97$ & 3.087 & 55 \\
\hline AAT $3.9 \mathrm{~m} / \mathrm{CYCLOPS}$ & 2015 Feb-May & 13 & 70 & $\cdots$ & 3.087 & 30 \\
\hline \multicolumn{7}{|l|}{ HATS-52 } \\
\hline ANU $2.3 \mathrm{~m} / \mathrm{WiFeS}$ & 2014 Jul 3 & 1 & 3 & 58 & $\cdots$ & $\cdots$ \\
\hline ANU $2.3 \mathrm{~m} / \mathrm{WiFeS}$ & 2014 Jul-2015 Jan & 4 & 7 & $14-50$ & 13.8 & 4000 \\
\hline Euler $1.2 \mathrm{~m} / \mathrm{CORALIE}$ & 2015 Mar 28 & 1 & 60 & 11 & 13.30 & $\cdots$ \\
\hline ESO 3.6 m/HARPS & 2015 Apr 6-8 & 3 & 115 & $10-13$ & 13.384 & 15 \\
\hline MPG $2.2 \mathrm{~m} /$ FEROS & 2015 Jun-2016 Feb & 11 & 48 & $25-48$ & 13.456 & 127 \\
\hline \multicolumn{7}{|l|}{ HATS-53 } \\
\hline ANU $2.3 \mathrm{~m} / \mathrm{WiFeS}$ & 2015 Mar 30 & 1 & 3 & 30 & $\cdots$ & $\ldots$ \\
\hline ANU $2.3 \mathrm{~m} / \mathrm{WiFeS}$ & 2015 Mar-Apr & 2 & 7 & $6-33$ & 68.5 & 4000 \\
\hline ESO 3.6 m/HARPS & 2015 Apr 7-8 & 2 & 115 & $7-12$ & 71.945 & 23 \\
\hline MPG $2.2 \mathrm{~m} /$ FEROS & 2015 Jun $6-20$ & 11 & 48 & $21-40$ & 71.950 & 28 \\
\hline
\end{tabular}

Notes.

${ }^{a} \mathrm{~S} / \mathrm{N}$ per resolution element near $5180 \AA$ A.

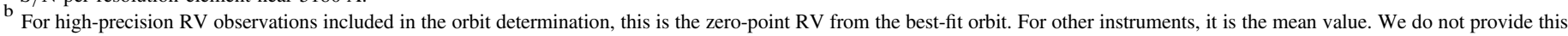

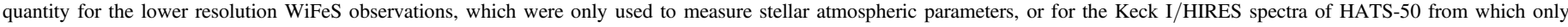
relative velocities have been measured.

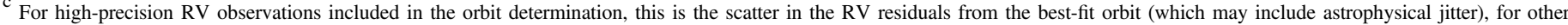

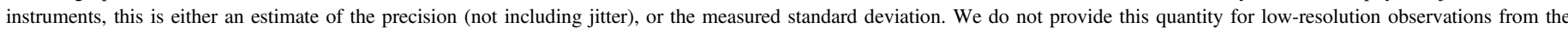
ANU $2.3 \mathrm{~m} / \mathrm{WiFeS}$.

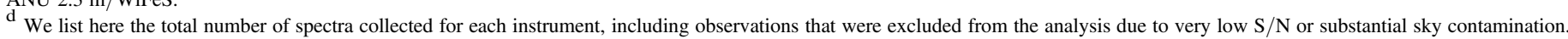
For HATS-50, we excluded one CORALIE spectrum and 5 FEROS spectra from the analysis. For HATS-51, we excluded 3 CORALIE spectra.
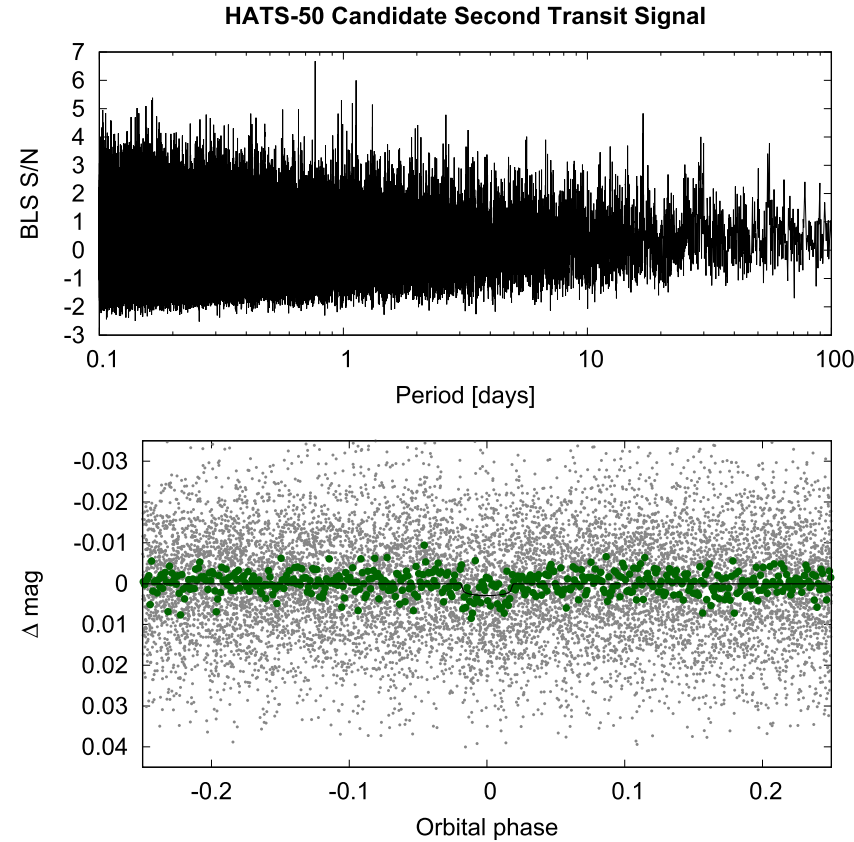

Figure 2. Search for additional periodic signals in the light curve of HATS-50 due to other transiting planets. Top panel: BLS spectrum. Bottom panel: unbinned light curve of HATS-50 (gray points) phase folded with the 0.76624822 days transit signal. The green points are the phase-binned values (bin size of 0.001). The line is a Mandel \& Agol (2002) transit-model fit to the light curve. long time-series sequence ( $>7000$ images) for a single field is collected, then aperture photometry is performed to get light curves for each star with $9 \lesssim r \lesssim 16$ mag in the field. The resulting light curves are treated with decorrelation and detrending algorithms $^{21}$ and, finally, we look for possible transiting-planet periodic signals by running the Box-fitting Least Squares (BLS; Kovács et al. 2002) code for each of them. Planet candidates detected from the survey undergo spectroscopic characterization and, finally, their planetary nature is confirmed or excluded by precise radial-velocity measurements and photometric follow-up observations (Penev et al. 2013).

Since first light in 2009, the HATSouth survey has so far produced 6.25 million light curves for 5.07 million stars from observations covering 2609 square degrees. This is due to the overlap between the pointing of the cameras on a single mount and between the different pointing positions we use to tile the sky into target fields. As a matter of fact, some stars have multiple light curves from different cameras and pointing positions (e.g., HATS-4: Jordán et al. 2014). Based on these observations, we have so far identified 1883 candidate transiting planets of which 1120 have undergone follow-up observations. This leads so far to the determination that 636 of the candidates are false alarms or false positives, while we confirmed and published 44 planets.

\footnotetext{
${ }^{21}$ External Parameter Decorrelation (EPD; Bakos et al. 2010); Trend Filtering Algorithm (TFA; Kovács et al. 2005).
} 
Table 3

Relative Radial Velocities and Bisector Spans for HATS-50-HATS-53

\begin{tabular}{|c|c|c|c|c|c|c|}
\hline $\begin{array}{l}\text { BJD } \\
(2450,000+)\end{array}$ & $\begin{array}{c}\mathrm{RV}^{\mathrm{a}} \\
\left(\mathrm{m} \mathrm{s}^{-1}\right)\end{array}$ & $\begin{array}{c}\sigma_{\mathrm{RV}}^{\mathrm{b}} \\
\left(\mathrm{m} \mathrm{s}^{-1}\right)\end{array}$ & $\begin{array}{c}\mathrm{BS} \\
\left(\mathrm{m} \mathrm{s}^{-1}\right)\end{array}$ & $\begin{array}{c}\sigma_{\mathrm{BS}} \\
\left(\mathrm{m} \mathrm{s}^{-1}\right)\end{array}$ & Phase & Instrument \\
\hline \multicolumn{7}{|l|}{ HATS-50 } \\
\hline 6828.86592 & -17.38 & 24.00 & 75.0 & 37.0 & 0.168 & Coralie \\
\hline 6829.74512 & 24.62 & 26.00 & 118.0 & 43.0 & 0.398 & Coralie \\
\hline 6857.78434 & 68.26 & 12.00 & 9.0 & 15.0 & 0.719 & FEROS \\
\hline 6858.73766 & 14.26 & 11.00 & 11.0 & 14.0 & 0.968 & FEROS \\
\hline 6859.76731 & -56.74 & 12.00 & 4.0 & 16.0 & 0.237 & FEROS \\
\hline $6862.68928^{d}$ & 144.26 & 33.00 & 50.0 & 41.0 & 0.000 & FEROS \\
\hline 6907.82693 & 58.01 & 4.95 & $\ldots$ & $\ldots$ & 0.786 & HIRES \\
\hline 6908.75386 & 31.96 & 5.67 & $\ldots$ & $\ldots$ & 0.028 & HIRES \\
\hline 6909.74379 & -4.97 & 11.27 & $\ldots$ & $\ldots$ & 0.287 & HIRES \\
\hline 6911.50060 & -62.38 & 41.00 & -301.0 & 63.0 & 0.746 & Coralie \\
\hline 6911.76564 & 49.40 & 4.49 & $\ldots$ & $\ldots$ & 0.815 & HIRES \\
\hline $6912.49456^{\mathrm{d}}$ & -62.38 & 30.00 & -178.0 & 51.0 & 0.005 & Coralie \\
\hline 6912.79856 & -36.69 & 4.79 & $\ldots$ & $\ldots$ & 0.084 & HIRES \\
\hline 6913.78648 & -68.78 & 5.12 & $\ldots$ & $\ldots$ & 0.342 & HIRES \\
\hline 6932.53890 & -176.74 & 13.00 & -75.0 & 17.0 & 0.239 & FEROS \\
\hline 6942.60598 & 0.26 & 13.00 & -29.0 & 16.0 & 0.868 & FEROS \\
\hline 6983.54362 & 67.26 & 11.00 & 23.0 & 15.0 & 0.557 & FEROS \\
\hline 7166.81632 & -64.74 & 15.00 & -30.0 & 19.0 & 0.413 & FEROS \\
\hline 7181.70212 & -3.74 & 14.00 & 8.0 & 18.0 & 0.300 & FEROS \\
\hline 7186.89313 & 112.26 & 18.00 & 75.0 & 24.0 & 0.655 & FEROS \\
\hline 7188.70636 & -22.74 & 13.00 & 15.0 & 18.0 & 0.129 & FEROS \\
\hline 7209.04868 & 1.46 & 6.60 & $\ldots$ & $\ldots$ & 0.440 & HIRES \\
\hline 7211.80755 & -45.74 & 12.00 & 21.0 & 16.0 & 0.161 & FEROS \\
\hline 7219.64476 & -47.74 & 12.00 & -19.0 & 17.0 & 0.207 & FEROS \\
\hline 7223.64984 & 6.26 & 15.00 & 36.0 & 20.0 & 0.253 & FEROS \\
\hline 7225.55693 & 62.26 & 11.00 & -11.0 & 15.0 & 0.751 & FEROS \\
\hline 7227.54760 & -73.74 & 13.00 & -41.0 & 17.0 & 0.271 & FEROS \\
\hline 7235.52005 & -191.74 & 34.00 & 99.0 & 20.0 & 0.352 & FEROS \\
\hline 7236.84001 & -114.74 & 17.00 & 129.0 & 19.0 & 0.697 & FEROS \\
\hline 7297.66592 & -52.74 & 18.00 & -177.0 & 24.0 & 0.580 & FEROS \\
\hline 7299.64213 & -83.74 & 16.00 & -16.0 & 22.0 & 0.096 & FEROS \\
\hline 7557.80118 & 62.26 & 12.00 & -28.0 & 17.0 & 0.505 & FEROS \\
\hline 7558.81095 & 80.26 & 12.00 & 33.0 & 17.0 & 0.769 & FEROS \\
\hline 7569.65730 & 106.26 & 15.00 & 93.0 & 22.0 & 0.601 & FEROS \\
\hline 7576.64710 & 41.26 & 16.00 & 39.0 & 21.0 & 0.426 & FEROS \\
\hline 7590.72922 & -25.94 & 10.50 & 22.0 & 14.0 & 0.103 & FEROS \\
\hline 7593.67838 & 4.86 & 12.90 & 54.0 & 17.0 & 0.874 & FEROS \\
\hline 7612.71943 & 113.86 & 12.30 & 6.0 & 17.0 & 0.845 & FEROS \\
\hline 7614.57198 & 79.66 & 12.60 & 127.0 & 16.0 & 0.329 & FEROS \\
\hline \multicolumn{7}{|l|}{ HATS-51 } \\
\hline 6939.83172 & -15.58 & 17.00 & -11.0 & 27.0 & 0.491 & Coralie \\
\hline 6940.81069 & 104.42 & 63.00 & 5.0 & 68.0 & 0.783 & Coralie \\
\hline 6941.86563 & -73.58 & 19.00 & -60.0 & 29.0 & 0.098 & Coralie \\
\hline 6967.78670 & 132.42 & 19.00 & 116.0 & 29.0 & 0.838 & Coralie \\
\hline 6969.79828 & 30.42 & 18.00 & 23.0 & 27.0 & 0.439 & Coralie \\
\hline 6972.69347 & -50.58 & 16.00 & 65.0 & 25.0 & 0.303 & Coralie \\
\hline 6997.69160 & 178.26 & 10.00 & 87.0 & 12.0 & 0.768 & FEROS \\
\hline 6997.73191 & 201.26 & 10.00 & 74.0 & 13.0 & 0.780 & FEROS \\
\hline 6999.63851 & -9.74 & 10.00 & 38.0 & 11.0 & 0.349 & FEROS \\
\hline 7030.81040 & 36.26 & 10.00 & 2.0 & 10.0 & 0.657 & FEROS \\
\hline 7033.73624 & 3.26 & 10.00 & 9.0 & 10.0 & 0.531 & FEROS \\
\hline 7035.75138 & -68.74 & 10.00 & -4.0 & 11.0 & 0.133 & FEROS \\
\hline 7036.67747 & -49.74 & 10.00 & 6.0 & 10.0 & 0.409 & FEROS \\
\hline 7037.79634 & 59.26 & 10.00 & 12.0 & 10.0 & 0.744 & FEROS \\
\hline 7049.60146 & -96.74 & 10.00 & -22.0 & 10.0 & 0.269 & FEROS \\
\hline 7050.67770 & 34.26 & 10.00 & 12.0 & 10.0 & 0.590 & FEROS \\
\hline 7053.74327 & -17.74 & 10.00 & -18.0 & 10.0 & 0.505 & FEROS \\
\hline 7054.60465 & 61.26 & 10.00 & -5.0 & 10.0 & 0.763 & FEROS \\
\hline 7055.64520 & -94.74 & 10.00 & -65.0 & 10.0 & 0.073 & FEROS \\
\hline 7057.74745 & 8.26 & 10.00 & -85.0 & 11.0 & 0.701 & FEROS \\
\hline 7075.63657 & -61.58 & 18.00 & 6.0 & 29.0 & 0.043 & Coralie \\
\hline 7076.65198 & -86.58 & 18.00 & -2.0 & 29.0 & 0.346 & Coralie \\
\hline
\end{tabular}


Table 3

(Continued)

\begin{tabular}{|c|c|c|c|c|c|c|}
\hline $\begin{array}{l}\text { BJD } \\
(2450,000+)\end{array}$ & $\begin{array}{c}\mathrm{RV}^{\mathrm{a}} \\
\left(\mathrm{m} \mathrm{s}^{-1}\right)\end{array}$ & $\begin{array}{c}\sigma_{\mathrm{RV}^{\mathrm{b}}} \\
\left(\mathrm{m} \mathrm{s}^{-1}\right)\end{array}$ & $\begin{array}{c}\mathrm{BS} \\
\left(\mathrm{m} \mathrm{s}^{-1}\right)\end{array}$ & $\begin{array}{c}\sigma_{\mathrm{BS}} \\
\left(\mathrm{m} \mathrm{s}^{-1}\right)\end{array}$ & Phase & Instrument \\
\hline 7078.63334 & -0.58 & 18.00 & -46.0 & 29.0 & 0.938 & Coralie \\
\hline 7080.99763 & 18.92 & 9.40 & $\ldots$ & $\ldots$ & 0.644 & CYCLOPS \\
\hline 7081.01370 & 42.82 & 6.40 & $\ldots$ & $\ldots$ & 0.649 & CYCLOPS \\
\hline 7081.07820 & 59.12 & 8.60 & $\ldots$ & $\ldots$ & 0.668 & CYCLOPS \\
\hline 7082.97896 & -58.38 & 9.00 & $\ldots$ & $\ldots$ & 0.235 & CYCLOPS \\
\hline 7082.99492 & -49.68 & 7.60 & $\cdots$ & $\cdots$ & 0.240 & CYCLOPS \\
\hline 7083.01087 & -34.78 & 11.70 & $\ldots$ & $\cdots$ & 0.245 & CYCLOPS \\
\hline 7149.86599 & -104.58 & 13.50 & $\ldots$ & $\ldots$ & 0.208 & CYCLOPS \\
\hline 7149.89323 & -100.98 & 13.00 & $\cdots$ & $\cdots$ & 0.217 & CYCLOPS \\
\hline 7149.90918 & -123.48 & 20.90 & $\ldots$ & $\cdots$ & 0.221 & CYCLOPS \\
\hline 7152.85935 & -57.48 & 12.10 & $\ldots$ & $\ldots$ & 0.102 & CYCLOPS \\
\hline 7152.86772 & -72.48 & 20.40 & $\ldots$ & $\cdots$ & 0.105 & CYCLOPS \\
\hline 7154.86321 & 77.12 & 5.30 & $\ldots$ & $\ldots$ & 0.701 & CYCLOPS \\
\hline 7154.87917 & 113.72 & 6.70 & $\ldots$ & $\ldots$ & 0.705 & CYCLOPS \\
\hline 7313.71457 & -4.58 & 18.00 & 147.0 & 49.0 & 0.135 & Coralie \\
\hline 7317.75708 & -228.58 & 19.00 & -74.0 & 38.0 & 0.342 & Coralie \\
\hline 7318.73072 & 53.42 & 18.00 & -27.0 & 38.0 & 0.633 & Coralie \\
\hline 7408.69120 & -28.58 & 12.00 & 16.0 & 20.0 & 0.496 & Coralie \\
\hline 7409.69015 & 14.42 & 12.00 & 10.0 & 20.0 & 0.794 & Coralie \\
\hline 7410.58904 & 82.42 & 14.00 & -92.0 & 23.0 & 0.063 & Coralie \\
\hline 7411.54533 & -88.58 & 19.00 & -141.0 & 32.0 & 0.348 & Coralie \\
\hline 7464.64412 & -70.58 & 17.00 & -54.0 & 32.0 & 0.204 & Coralie \\
\hline 7466.52399 & 82.42 & 17.00 & 25.0 & 29.0 & 0.765 & Coralie \\
\hline 7640.86434 & 63.42 & 16.00 & 23.0 & 29.0 & 0.825 & Coralie \\
\hline 7643.82622 & 61.42 & 13.00 & -23.0 & 26.0 & 0.709 & Coralie \\
\hline 7645.89470 & -50.58 & 12.00 & 4.0 & 22.0 & 0.327 & Coralie \\
\hline \multicolumn{7}{|l|}{ HATS-52 } \\
\hline 7109.65449 & -323.80 & 34.00 & $\ldots$ & $\ldots$ & 0.165 & Coralie \\
\hline 7118.61984 & 378.20 & 24.00 & -15.0 & 31.0 & 0.725 & HARPS \\
\hline 7119.61676 & -82.80 & 31.00 & 95.0 & 37.0 & 0.455 & HARPS \\
\hline 7120.59836 & -341.80 & 28.00 & -8.0 & 37.0 & 0.173 & HARPS \\
\hline 7181.53193 & 324.38 & 24.00 & -136.0 & 24.0 & 0.759 & FEROS \\
\hline 7185.51437 & 228.38 & 25.00 & -24.0 & 24.0 & 0.673 & FEROS \\
\hline 7186.48111 & -235.62 & 22.00 & 125.0 & 21.0 & 0.380 & FEROS \\
\hline 7187.48351 & -333.62 & 25.00 & -36.0 & 24.0 & 0.114 & FEROS \\
\hline 7190.47681 & -355.62 & 24.00 & 53.0 & 24.0 & 0.304 & FEROS \\
\hline 7324.83438 & 566.38 & 19.00 & 146.0 & 18.0 & 0.615 & FEROS \\
\hline 7325.84444 & -219.62 & 15.00 & 86.0 & 15.0 & 0.354 & FEROS \\
\hline 7327.85022 & 386.38 & 18.00 & 137.0 & 18.0 & 0.822 & FEROS \\
\hline 7403.83591 & -196.62 & 15.00 & 16.0 & 15.0 & 0.422 & FEROS \\
\hline 7405.78818 & 330.38 & 17.00 & 33.0 & 17.0 & 0.850 & FEROS \\
\hline 7447.77082 & -15.62 & 18.00 & -107.0 & 18.0 & 0.570 & FEROS \\
\hline \multicolumn{7}{|l|}{ HATS-53 } \\
\hline 7119.69799 & 57.38 & 45.00 & -77.0 & 53.0 & 0.625 & HARPS \\
\hline 7120.69688 & 86.38 & 19.00 & -83.0 & 29.0 & 0.884 & HARPS \\
\hline 7181.59141 & 72.23 & 16.00 & 54.0 & 21.0 & 0.686 & FEROS \\
\hline 7182.52590 & 28.23 & 14.00 & -17.0 & 18.0 & 0.928 & FEROS \\
\hline 7183.59764 & -86.77 & 14.00 & 13.0 & 18.0 & 0.206 & FEROS \\
\hline 7184.48225 & -9.77 & 15.00 & 15.0 & 19.0 & 0.436 & FEROS \\
\hline 7185.62553 & 103.23 & 21.00 & -43.0 & 28.0 & 0.732 & FEROS \\
\hline 7186.57088 & 62.23 & 18.00 & -63.0 & 24.0 & 0.978 & FEROS \\
\hline 7187.63960 & -48.77 & 18.00 & -63.0 & 24.0 & 0.255 & FEROS \\
\hline 7190.53242 & -32.77 & 15.00 & -65.0 & 19.0 & 0.006 & FEROS \\
\hline 7192.50171 & -36.77 & 21.00 & -98.0 & 28.0 & 0.517 & FEROS \\
\hline 7193.61040 & 82.23 & 15.00 & 6.0 & 19.0 & 0.804 & FEROS \\
\hline 7194.49223 & -27.77 & 14.00 & 16.0 & 17.0 & 0.033 & FEROS \\
\hline
\end{tabular}

Notes.

${ }^{\text {a }}$ The zero-point of these velocities is arbitrary. An overall offset $\gamma_{\text {rel }}$ fitted independently to the velocities from each instrument has been subtracted.

${ }^{\mathrm{b}}$ Internal errors excluding the component of astrophysical jitter are considered in Section 3.3.

(This table is available in machine-readable form.) 

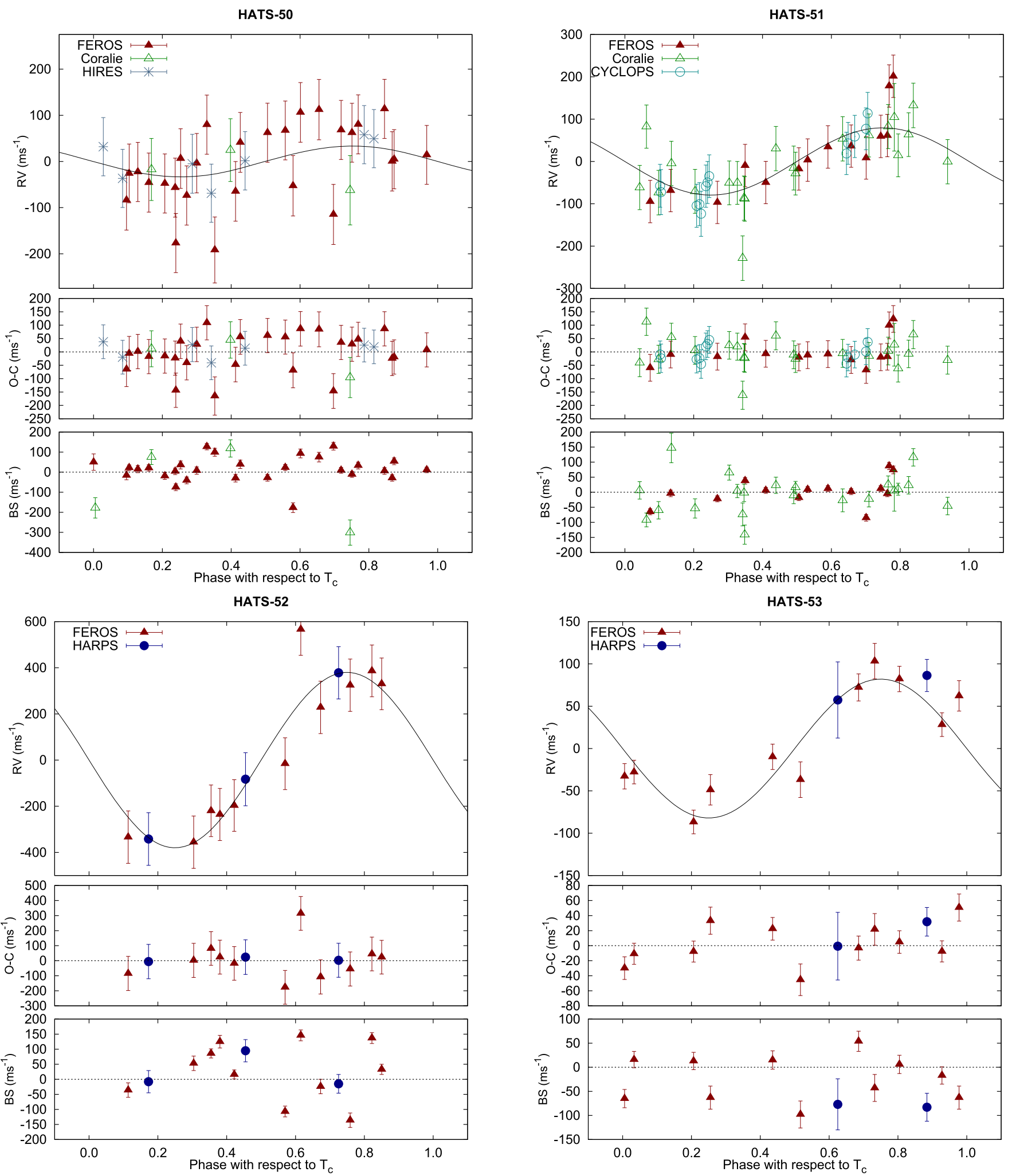

Figure 3. Phased high-precision RV measurements for HATS-50 (upper left), HATS-51 (upper right), HATS-52 (lower left), and HATS-53 (lower right). The instruments used are labeled in the plots. In each case, we show three panels. The top panel shows the phased measurements together with our best-fit model (see Table 6) for each system. Zero-phase corresponds to the time of mid-transit. The center-of-mass velocity has been subtracted. The second panel shows the velocity $O-C$ residuals from the best fit. The error bars include the jitter terms listed in Table 6 added in quadrature to the formal errors for each instrument. The third panel shows the bisector spans (BS). Note the different vertical scales of the panels.

Notable cases are: the two super-Neptunes HATS-7b (Bakos et al. 2015) and HATS-8b (Bayliss et al. 2015); HATS-6b, a warm Saturn-mass exoplanet orbiting an M star (Hartman et al.
2015); HATS-17b, the longest period transiting exoplanet discovered so far by a wide-field ground-based photometric survey (Brahm et al. 2016); HATS-18b, an extremely short- 
Table 4

Light Curve Data for HATS-50, HATS-51, HATS-52, and HATS-53

\begin{tabular}{|c|c|c|c|c|c|c|}
\hline Object $^{\mathrm{a}}$ & $\begin{array}{c}\text { BJD }^{\mathrm{b}} \\
(2400,000+)\end{array}$ & $\mathrm{Mag}^{\mathrm{c}}$ & $\sigma_{\mathrm{Mag}}$ & $\operatorname{Mag}\left(\right.$ orig) ${ }^{\mathrm{d}}$ & Filter & Instrument \\
\hline HATS-50 & 55451.44267 & 0.00058 & 0.00718 & $\cdots$ & $r$ & HS/G580.4 \\
\hline HATS-50 & 55765.47934 & 0.00588 & 0.00716 & $\cdots$ & $r$ & HS/G580.4 \\
\hline HATS-50 & 55788.45820 & 0.00832 & 0.00928 & $\cdots$ & $r$ & HS/G580.4 \\
\hline HATS-50 & 55516.54955 & -0.00368 & 0.01095 & $\ldots$ & $r$ & HS/G580.4 \\
\hline HATS-50 & 55478.25269 & -0.01940 & 0.00810 & $\ldots$ & $r$ & HS/G580.4 \\
\hline HATS-50 & 55451.44609 & -0.01299 & 0.00728 & $\ldots$ & $r$ & HS/G580.4 \\
\hline HATS-50 & 55738.67412 & -0.00224 & 0.00857 & $\cdots$ & $r$ & HS/G580.4 \\
\hline HATS-50 & 55470.59492 & -0.00388 & 0.00781 & $\ldots$ & $r$ & HS/G580.4 \\
\hline HATS-50 & 55765.48280 & -0.00187 & 0.00699 & $\cdots$ & $r$ & HS/G580.4 \\
\hline HATS-50 & 55516.55298 & 0.01526 & 0.01119 & $\ldots$ & $r$ & HS/G580.4 \\
\hline
\end{tabular}

Notes.

${ }^{\text {a }}$ Either HATS-50, HATS-51, HATS-52, or HATS-53.

b Barycentric Julian Date is computed directly from the UTC time without correction for leap seconds.

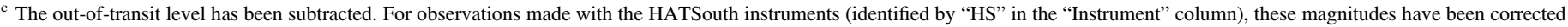

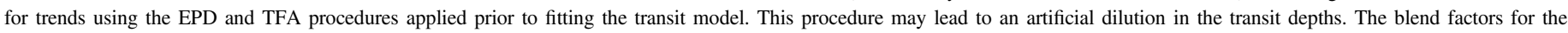

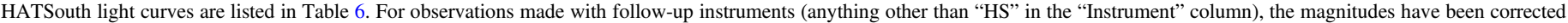
for a quadratic trend in time, and for variations correlated with up to three PSF shape parameters, fit simultaneously with the transit.

${ }^{\mathrm{d}}$ Raw magnitude values without correction for the quadratic trend in time, or for trends correlated with the seeing. These are only reported for the follow-up observations.

(This table is available in its entirety in machine-readable form.)

period planet spinning-up its host star (Penev et al. 2016); the detection of several very low-mass stars $\left(0.1-0.2 M_{\odot}\right)$ in eclipsing binary systems (Zhou et al. 2014, 2015).

Currently, dozens of other exoplanets have been confirmed by the HATSouth team and are undergoing analysis and preparation for publication.

\subsection{Photometric Detection}

This study presents the discovery of four new transiting planetary systems, which were detected following the procedure described above, and confirmed based on follow-up observations as described in the next sections; the new systems are HATS-50, HATS-51, HATS-52, and HATS-53. Each of them are composed of a moderately bright G-type star and a hot-Jupiter-type planet. The orbital periods are 3.8297 days, 3.3489 days, 1.3667 days, and 3.8538 days for HATS-50b, HATS-51b, HATS-52b, and HATS-53b, respectively, implying that we are dealing with new close-in hot Jupiters. Stellar coordinates, magnitudes, and cross-identifications are shown in Table 5. In particular, the magnitudes of the fours stars in the optical bands were taken from APASS (Henden et al. 2009), as listed in the UCAC 4 catalog (Zacharias et al. 2012), while those in the NIR bands are from the 2MASS catalog.

A summary of the HATSouth photometric observations for these objects is reported in Table 1. In particular, the four stars were observed thousands of times by the HATSouth telescopes between 2010 March and 2013 July; the corresponding phasefolded light curves are plotted in Figure 1, clearly showing typical transiting-planet signals with transit depths around $1 \%-2 \%$. The data concerning all photometric observations are available in electronic format in Table 4.

\subsection{Searching for Additional Periodic Signals in the Time-series Survey Data}

After having detected the planetary signals, we further analyze each of the four data sets to search for potential stellar variability/activity and additional periodic signals due to other transiting planets. This analysis was carried out by running BLS on the residuals of each HATSouth light curve and studying the Generalized Lomb-Scargle periodogram (GLS; Zechmeister \& Kürster 2009). The results of these additional checks are as follows:

1. By running BLS, we did not detected any significant periodic transit signal in the residuals of the HATS-51, HATS-52, and HATS-53 light curves. For the case of HATS-50, we noticed a marginal transit signal with a period of 0.76624822 days, $T_{\mathrm{C}}=2455274.38586$, depth of $3.2 \mathrm{mmag}$, and duration of 46 minutes (bottom panel of Figure 2). The candidate transit signal has a signal-tonoise ratio $(\mathrm{S} / \mathrm{N})$ of 7.5 in the phase-folded light curve and a BLS Signal-detection-efficiency (SDE) value of 7.38. Even though this signal is below our threshold for selecting candidates to follow up (top panel of Figure 2), it is worth noting given the presence of the confirmed hot Jupiter and the possibility of close companions to hot Jupiters (Becker et al. 2015). Therefore, HATS-50 could be an interesting target for a further short-cadence, timeseries photometric monitoring with a more precise instrument, like TESS (Ricker \& TESS Science Team 2017). However, given the density of the host star HATS-50 inferred from modeling the transits of HATS-50b (see Table 5), and the duration of the transits for the candidate signal, the candidate planet would have an orbital inclination that differs by more than $10^{\circ}$ from that of the hot Jupiter. We finally note that a Mandel \& Agol (2002) model fit indicates an implied radius in the super-Neptune regime. So, if this signal was really caused by an additional transiting planet, it would be firmly in the Neptune desert.

2. By running GLS, we did not detect any significant periodic signal in the GLS spectrum of the light curve obtained for HATS-50, HATS-51, and HATS-53. For these three systems, we placed a $95 \%$ confidence upper limit of $0.95 \mathrm{mmag}$ on the amplitude of any periodic signal between 0.01 days and 100 days. For HATS-52, 
HATS-50

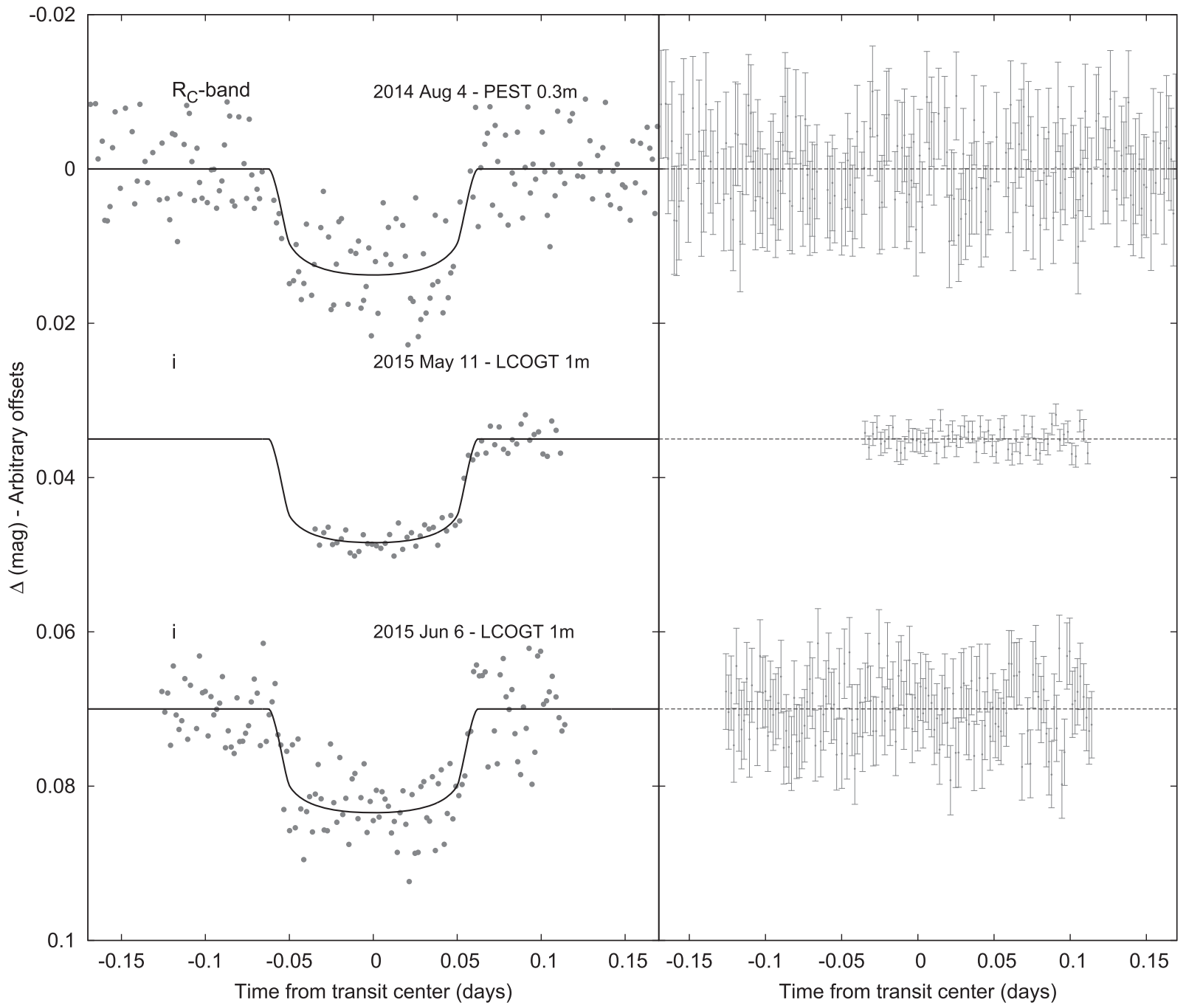

Figure 4. Unbinned transit light curves for HATS-50. The light curves have been corrected for quadratic trends in time, and linear trends with up to three parameters characterizing the shape of the PSF, fitted simultaneously with the transit model. The dates of the events, filters, and instruments used are indicated. Light curves following the first are displaced vertically for clarity. Our best fit from the global modeling described in Section 3.3 is shown by the solid lines. The residuals from the best-fit model are shown on the right-hand side in the same order as the original light curves. The error bars represent the photon and background shot noise, plus the readout noise.

we detected a sinusoidal signal with a periodicity of $P=15.63703 \pm 0.00066$ days and an amplitude of $1.23 \pm 0.25 \mathrm{mmag}$. The peak has an $\mathrm{S} / \mathrm{N}$ of 20.3 in the spectrum, a periodogram value of $\Delta \chi^{2} / \chi_{0}^{2}=0.0054$, and a false alarm probability (FAP), assuming Gaussian white noise, of $2 \times 10^{-5}$. We also assessed the FAP of GLS by performing a bootstrap analysis and obtaining a distribution of peak signals. From this, we estimated a more accurate FAP of $9 \times 10^{-5}$.

This sinusoidal periodic signal can be related to the stellar activity and, therefore, presumably indicates its rotation period. However, considering that HATS-52 has a radius of $R_{\star}=1.046 \pm 0.058 R_{\odot}$ (see Table 5), this value of $P$ implies that $v_{\mathrm{eq}}=3.60 \pm 0.59 \mathrm{~km} \mathrm{~s}^{-1}$ for HATS-52, which is $1.5 \sigma$ below the spectroscopically determined value of $v \sin i=4.59 \pm 0.64 \mathrm{~km} \mathrm{~s}^{-1}$. So, there is a slight tension between these measurements if we identify the photometric periodicity of $P=15.6$ days with the rotation period of the star.

\subsection{Spectroscopic Observations}

The first step that was undertaken in confirming the planetary nature of the four planetary candidates was to obtain a spectral reconnaissance of their host stars. This allows us to rule out the usual false positive cases (giant stars, binary systems, and blending with faint eclipsing binary systems). For this purpose, we used the Wide Field Spectrograph (WiFeS; Dopita et al. 2007), mounted on the ANU $2.3 \mathrm{~m}$ telescope at SSO. The spectroscopic parameters were estimated by taking low-resolution spectra $(R=3000)$. All four targets were identified as dwarf stars. We also took medium-resolution spectra $(R=7000)$, with the aim to search for possible $\mathrm{RV}$ variations at the $\sim 2 \mathrm{~km} \mathrm{~s}^{-1}$ level, which are useful to rule out possible stellar companions. Details about the data reduction and the processing of the $\mathrm{WiFeS}$ spectra are summarized in Bayliss et al. (2013).

Precise RV measurements of the targets were then acquired by using several medium- and large-class telescopes, equipped 


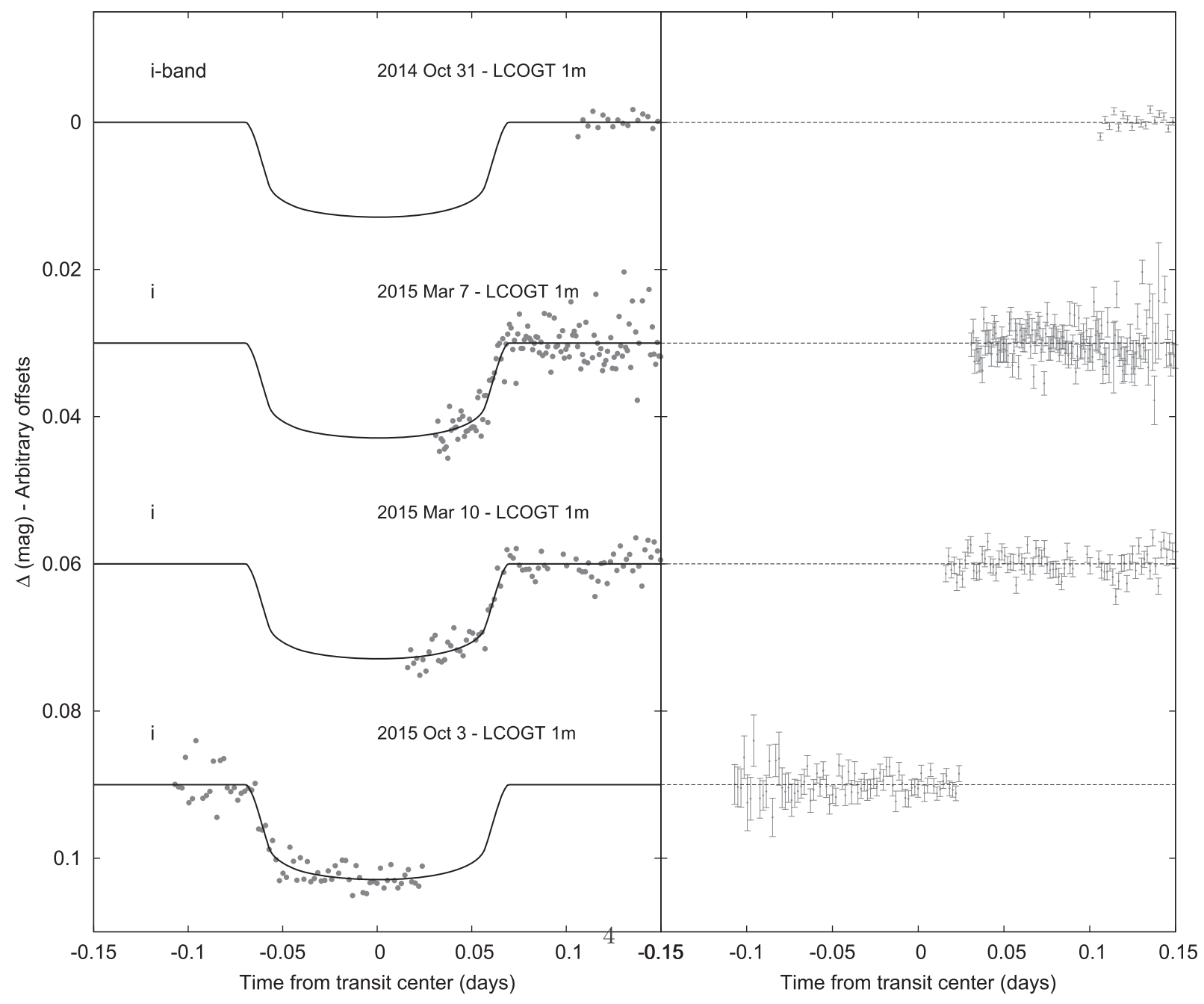

Figure 5. Same as Figure 4; here we show light curves for HATS-51.

with high-resolution spectrographs and working on wide ranges of optical wavelengths. They are summarized in Table 2, together with their main characteristics. With these instruments, it was possible to measure periodic RV variation of the stars, which is compatible with the presence of planettype objects orbiting around them. In particular, we mainly used the FEROS spectrograph (Kaufer \& Pasquini 1998), which is mounted on the MPG $2.2 \mathrm{~m}$ telescope at the ESO Observatory in La Silla, for monitoring the four targets. Other spectra were collected thanks to CORALIE (Queloz et al. 2001) on the Euler $1.2 \mathrm{~m}$ telescope, HARPS (Mayor et al. 2003) on the ESO $3.6 \mathrm{~m}$ telescope, which are also located at the La Silla observatory, and CYCLOPS mounted on the $3.9 \mathrm{~m}$ Anglo-Australian Telescope at SSO. For the case of HATS-50, which is the faintest star of the four $(V=14.0 \mathrm{mag})$, we needed higher $\mathrm{S} / \mathrm{N}$ measurements. These were obtained by taking seven spectra with the HIRES spectrograph (Vogt et al. 1994) on the Keck I $10 \mathrm{~m}$ telescope at Mauna Kea Observatory in Hawaii.

More details about the instruments, the data reduction, and the computation of the corresponding RVs can be found in the previous works of the HATSouth team, e.g., Penev et al. (2013),
Mohler-Fischer et al. (2003), and Bayliss et al. (2013). In particular, HARPS, FEROS, and CORALIE spectra were analyzed with the method described in Jordán et al. (2014) and Brahm et al. (2017a), while those coming from CYCLOPS in Addison et al. (2013). Finally, we refer the reader to Bakos et al. (2015) and Howard et al. (2010) for the analysis of the Keck/HIRES spectra.

The values of the RV measurements are reported in Table 3, while the phased RVs and bisector spans (BS) are plotted for each system in Figure 3. We also used the FEROS highresolution spectra for an accurate determination of the stellar spectroscopic parameters (effective temperature, metal abundance, and projected rotational velocity) by applying the Zonal Atmospherical Stellar Parameter Estimator (ZASPE) routine (Brahm et al. 2017b). This analysis is discussed in Section 3.1.

\subsection{Photometric Follow-up Observations}

Another important step for confirming and characterizing a transiting exoplanetary system consists of performing photometric follow-up observations of transit events. We can thus derive more precise measurements, with respect to the survey data, of the transit depth, duration, mid-transit time, and contact points of the corresponding light curves. An accurate 
HATS-52

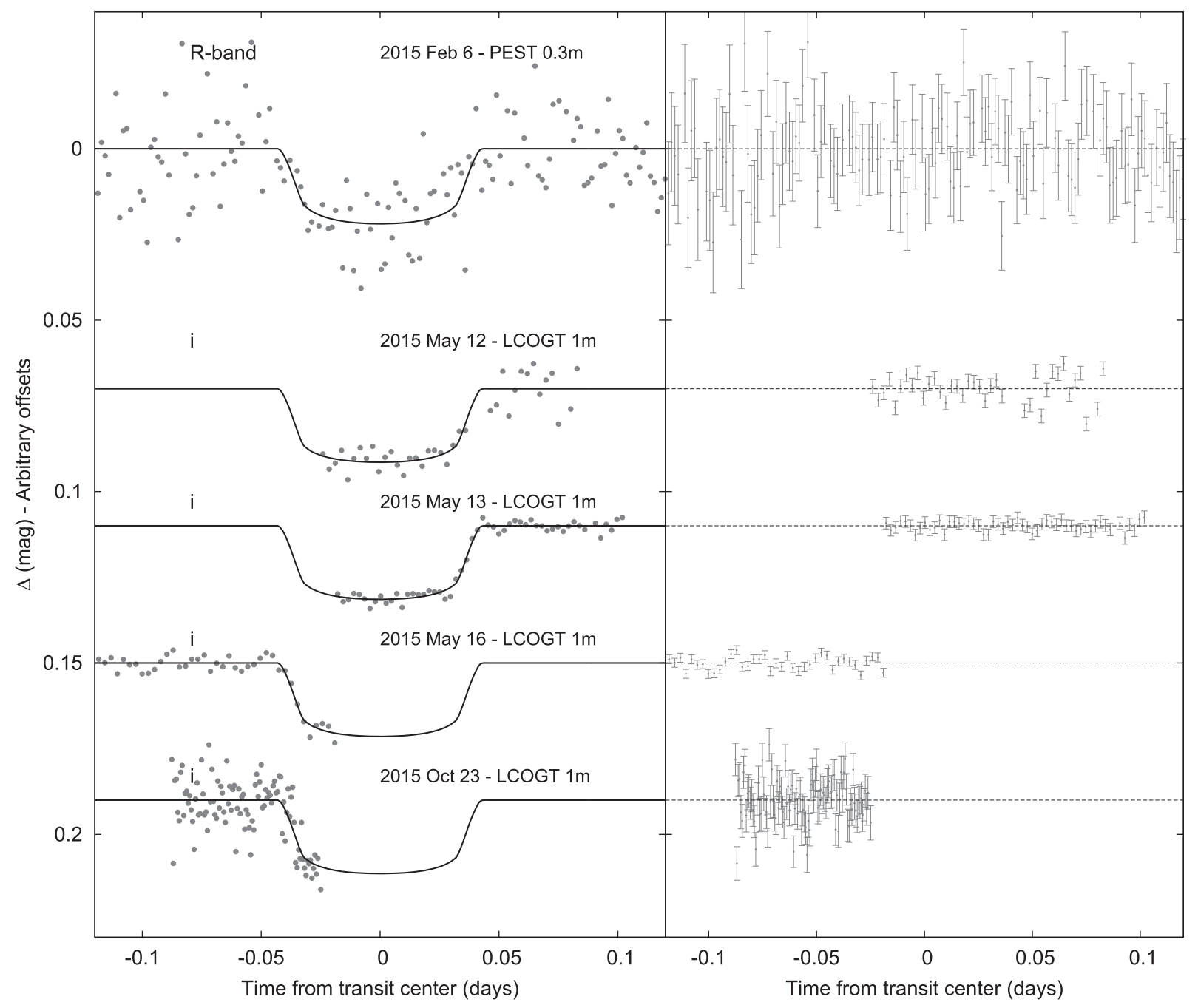

Figure 6. Same as Figure 4; here we show light curves for HATS-52.

knowledge of these photometric parameters are vital for robustly constraining the orbital parameters of the system and the physical parameters of both the star and the planet.

One complete transit event was observed with the PEST $0.3 \mathrm{~m}$ telescope ${ }^{22}$ for HATS-50b, HATS-52b, and HATS-53b through an $R$-band filter. Details of this telescope and the method used for reducing the data are described in Zhou et al. (2014). The other 11 transit light curves of the four targets were recorded using the $1 \mathrm{~m}$ telescopes (CTIO, SAAO, SSO) in the Las Cumbres Observatory Global Telescope network (LCOGT; Brown et al. 2013) and Sloan- $i^{\prime}$ filters. The LCOGT telescopes and the corresponding data reduction are described in Hartman et al. (2015). An excerpt of these observations is reported in Table 1. The light curves are plotted in Figures 4-7, for HATS-50, HATS-51, HATS-52, and HATS-53, respectively, and are compared to our best-fit models.

\subsection{Lucky Imaging}

Lucky imaging observations were obtained through a $z^{\prime}$ filter for the HATS-51 and HATS-52 systems using the Astralux Sur

\footnotetext{
${ }^{22}$ http://pestobservatory.com
}

camera (Hippler et al. 2009) on the New Technology Telescope (NTT) at La Silla Observatory in Chile, on the nights of 2015 December 22 and 23. Observations with this facility were carried out and reduced following Espinoza et al. (2016), but a plate scale of 15.20 mas pixel $^{-1}$ (derived in the work of Janson et al. 2017) was used. Figure 8 shows the reduced final images for each system, while Figure 9 shows the contrast curves based on these images produced using the technique and software described in Espinoza et al. (2016).

For HATS-52, a neighboring source is clearly detected at a distance of $2.74 \pm 0.03$ arcsec to the east and $0.79 \pm 0.03$ arcsec to the south from the target (i.e., at a distance of $2.85 \pm 0.03$ arcsec from the target), with $\Delta z^{\prime}=2.457 \pm 0.013 \mathrm{mag}$, relative to the target. Based on the photometric follow-up observations of this system that were carried out with the LCOGT $1 \mathrm{~m}$ telescope network (Section 2.5), we were able to determine that the transits occur around the star HATS-52 and not the neighbor. The final combined image has an effective FWHM of 0 " $0722 \pm 0$ ! 0050 . The same source was detected by the GAIA space observatory (GAIA Data Release 1; Lindegren et al. 2016) at $\approx 2$ ". 8 separation to the East from HATS-52, with $\Delta G_{\mathrm{GAIA}}=2.26$. Closer sources were not detected. 
HATS-53

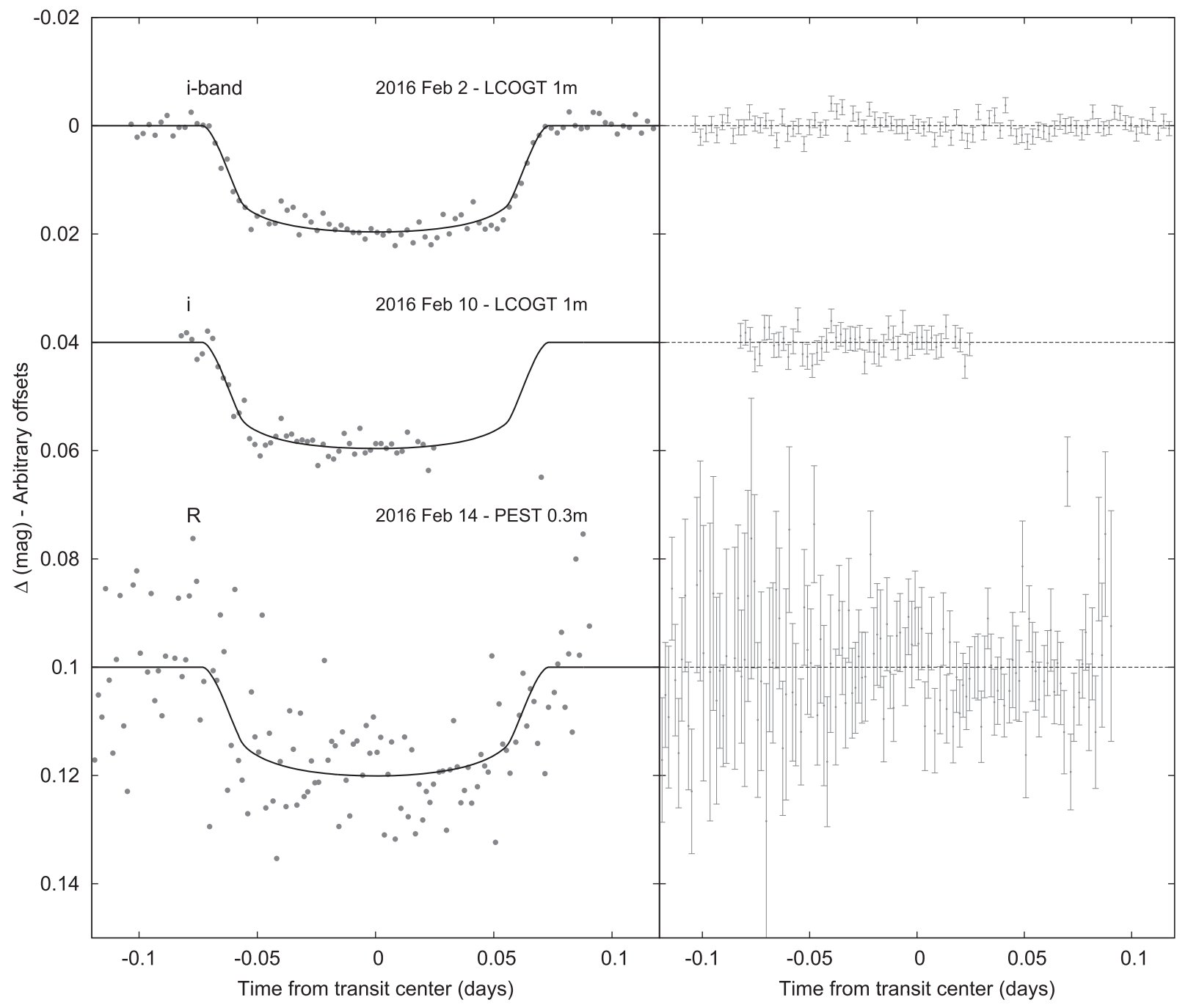

Figure 7. Same as Figure 4; here we show light curves for HATS-53.
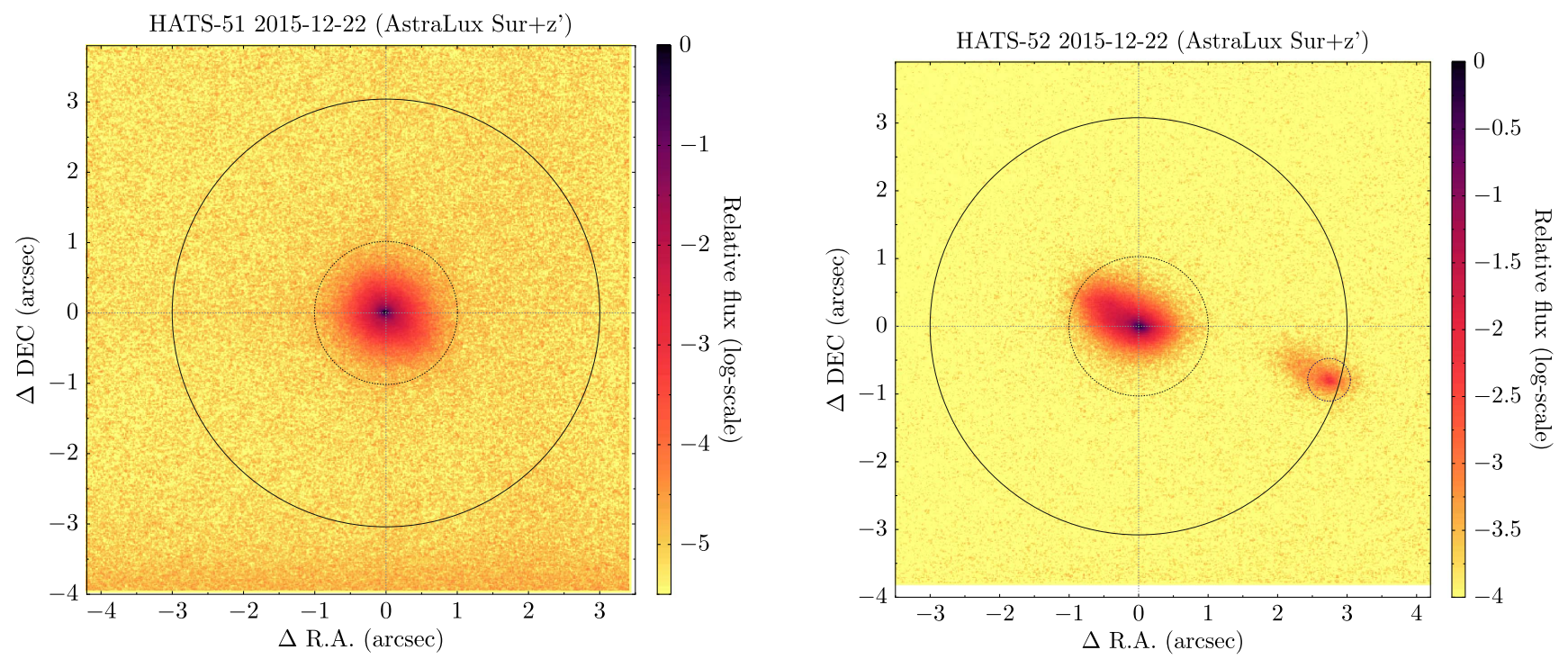

Figure 8. Astralux lucky images of HATS-51 (left) and HATS-52 (right). No neighboring source is detected for HATS-51. For HATS-52, a neighbor is clearly detected at $\Delta$ R.A. $\approx 3^{\prime \prime}, \Delta$ decl. $\approx-1^{\prime \prime}$ and with $\Delta z^{\prime}=2.457 \pm 0.013$ mag. 

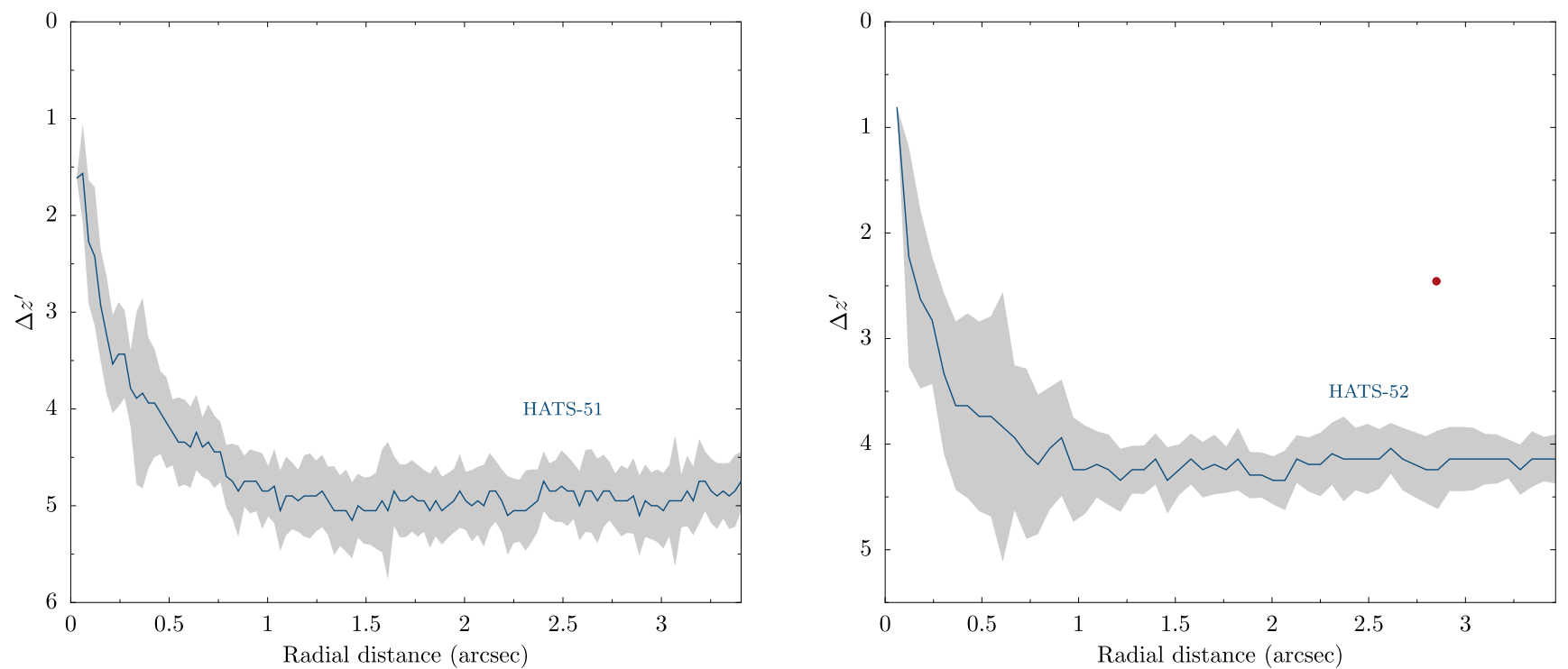

Figure 9. Contrast curves for HATS-51 (left) and HATS-52 (right) based on our AstraLux Sur $z^{\prime}$-band observations. Gray bands show the uncertainty given by the scatter in the contrast in the azimuthal direction at a given radius. The neighbor to HATS-52 is marked with the red filled circle.
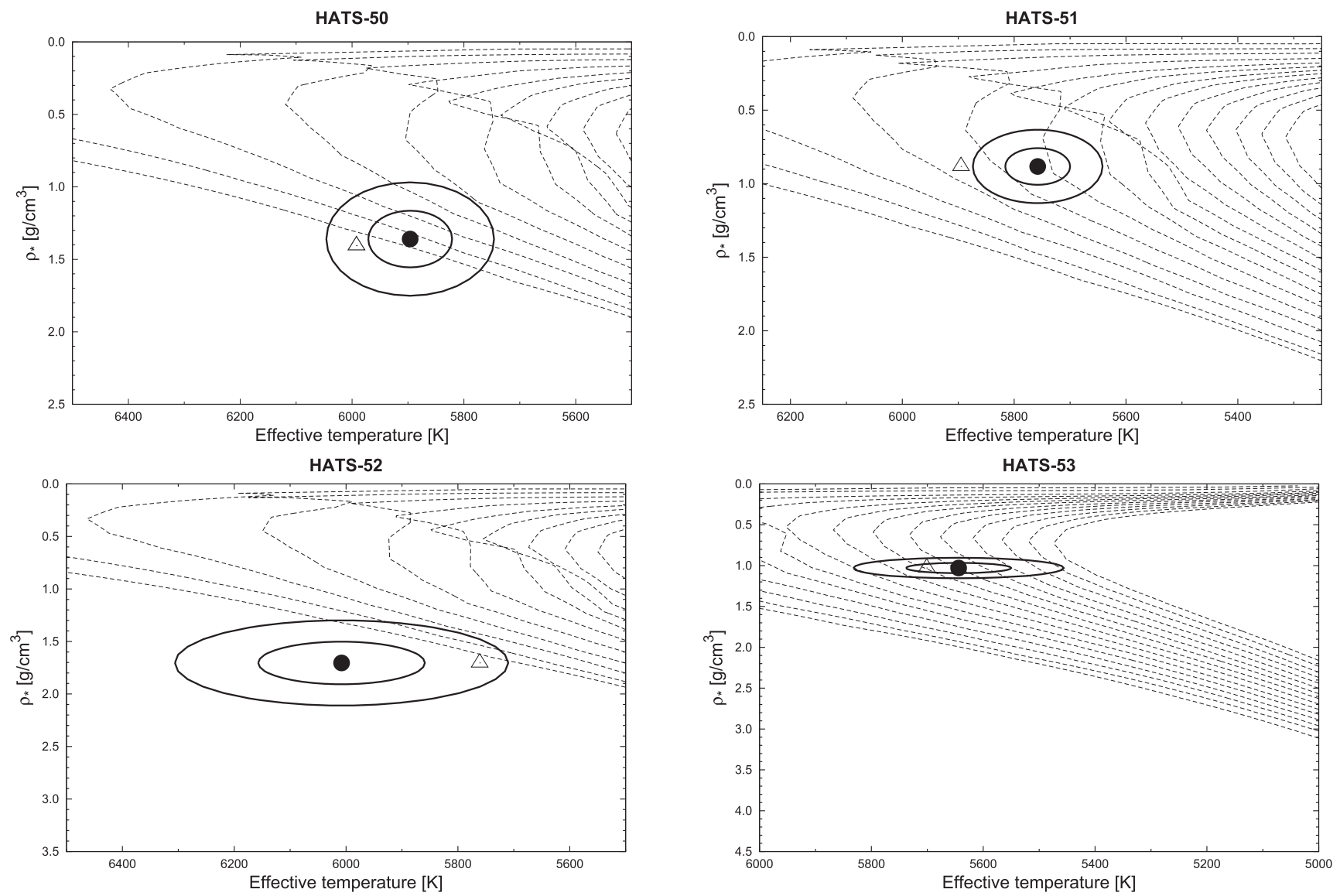

Figure 10. Model isochrones from Yi et al. (2001) for the measured metallicities of HATS-50 (upper left), HATS-51 (upper right), HATS-52 (lower left), and HATS53 (lower right). We show models for ages of $0.2 \mathrm{Gyr}$ and $1.0-14.0 \mathrm{Gyr}$ in $1.0 \mathrm{Gyr}$ increments (ages increasing from left to right). The adopted values of $T_{\text {eff }}$ and $\rho_{\star}$ are shown together with their $1 \sigma$ and $2 \sigma$ confidence ellipsoids. The initial values of $T_{\text {eff } \star}$ and $\rho_{\star}$ from the first ZASPE and light curve analyses of HATS-50 and HATS-53 are represented with open triangles.

For HATS-51, no neighbors were detected with the Astralux Sur camera (effective FWHM of $0 !^{\prime \prime} 0431 \pm 0$. "0053) and neither with GAIA within $10^{\prime \prime}$. Instead, based on GAIA data, we report that HATS-50 has a neighbor at 2 ". 1 to the east $(\Delta G=2.97)$, while HATS-53 has no neighbors within 8" 0 .

\section{Analysis}

Here, we describe the analysis of the observational data, which were presented in the previous section, with the aim to obtain complete physical characterizations of the HATS-50, HATS-51, HATS-52, and HATS-53 planetary systems. 
Table 5

Stellar Parameters for HATS-50-HATS-53

\begin{tabular}{|c|c|c|c|c|c|}
\hline Parameter & $\begin{array}{l}\text { HATS-50 } \\
\text { Value }\end{array}$ & $\begin{array}{l}\text { HATS-51 } \\
\text { Value }\end{array}$ & $\begin{array}{l}\text { HATS-52 } \\
\text { Value }\end{array}$ & $\begin{array}{l}\text { HATS-53 } \\
\text { Value }\end{array}$ & Source \\
\hline \multicolumn{6}{|c|}{$\begin{array}{l}\text { Astrometric Properties and Cross- } \\
\text { identifications }\end{array}$} \\
\hline 2MASS-ID & 20014273-2604392 & 06512340-2903309 & 09202105-3116095 & $11463084-3351361$ & \\
\hline GSC-ID & GSC 6896-01012 & GSC 6534-00607 & GSC 7153-01785 & GSC 7225-00413 & \\
\hline R.A. (J2000) & $20^{\mathrm{h}} 01^{\mathrm{m}} 42^{\mathrm{s}} \cdot 60$ & $06^{\mathrm{h}} 51^{\mathrm{m}} 23^{\mathrm{s}} .40$ & $09^{\mathrm{h}} 20^{\mathrm{m}} 21^{\mathrm{s}} .05$ & $11^{\mathrm{h}} 46^{\mathrm{m}} 30^{\mathrm{s}} .72$ & 2MASS \\
\hline Decl. (J2000) & $-26^{\circ} 04^{\prime} 39^{\prime \prime} 3$ & $-29^{\circ} 03^{\prime} 31^{\prime \prime} 0$ & $-31^{\circ} 16^{\prime} 09^{\prime \prime} 6$ & $-33^{\circ} 51^{\prime} 36^{\prime \prime} .2$ & 2MASS \\
\hline$\mu_{\text {R.A. }}\left({\left.\operatorname{mas~} \mathrm{yr}^{-1}\right)}^{-1}\right.$ & $3.2 \pm 1.6$ & $15.5 \pm 1.2$ & $27.7 \pm 3.7$ & $35.0 \pm 2.0$ & UCAC4 \\
\hline$\mu_{\text {Decl. }}\left({\left.\operatorname{mas~} \mathrm{yr}^{-1}\right)}^{-1}\right.$ & $1.6 \pm 1.6$ & $6.9 \pm 1.1$ & $16.0 \pm 3.7$ & $5.0 \pm 2.1$ & UCAC4 \\
\hline \multicolumn{6}{|c|}{ Spectroscopic Properties } \\
\hline$T_{\text {eff } *}(\mathrm{~K})$ & $5990 \pm 110$ & $5758 \pm 58$ & $6010 \pm 150$ & $5644 \pm 94$ & ZASPE $^{\mathrm{a}}$ \\
\hline$[\mathrm{Fe}=\mathrm{H}]$ & $0.300 \pm 0.056$ & $0.300 \pm 0.030$ & $0.22 \pm 0.10$ & $0.010 \pm 0.066$ & ZASPE \\
\hline$v \sin i\left(\mathrm{~km} \mathrm{~s}^{-1}\right)$ & $3.76 \pm 0.54$ & $3.98 \pm 0.26$ & $4.59 \pm 0.64$ & $2.50 \pm 0.76$ & ZASPE \\
\hline$v_{\text {mac }}\left(\mathrm{km} \mathrm{s}^{-1}\right)$ & $4.32 \pm 0.17$ & $3.962 \pm 0.088$ & $4.35 \pm 0.23$ & $3.79 \pm 0.14$ & Assumed \\
\hline$v \operatorname{mic}\left(\mathrm{km} \mathrm{s}^{-1}\right)$ & $1.225 \pm 0.085$ & $1.070 \pm 0.034$ & $1.24 \pm 0.12$ & $1.006 \pm 0.049$ & Assumed \\
\hline$\gamma_{\mathrm{RV}}\left(\mathrm{m} \mathrm{s}^{-1}\right)$ & $-20250 \pm 13$ & $3093 \pm 15$ & $13456 \pm 43$ & $71949.5 \pm 7.1$ & FEROS $^{\mathrm{b}}$ \\
\hline \multicolumn{6}{|c|}{ Photometric Properties } \\
\hline$G(\mathrm{mag})$ & 13.8 & 12.24 & 13.54 & 13.57 & GAIA DR $1^{\mathrm{c}}$ \\
\hline$B(\mathrm{mag})$ & $14.718 \pm 0.010$ & $13.190 \pm 0.030$ & $14.316 \pm 0.030$ & $14.548 \pm 0.040$ & APASS $^{\mathrm{d}}$ \\
\hline$V(\mathrm{mag})$ & $14.033 \pm 0.050$ & $12.471 \pm 0.030$ & $13.669 \pm 0.040$ & $13.790 \pm 0.030$ & APASS $^{\mathrm{d}}$ \\
\hline$g(\mathrm{mag})$ & $\ldots$ & $12.766 \pm 0.030$ & $13.962 \pm 0.020$ & $14.137 \pm 0.020$ & APASS $^{\mathrm{d}}$ \\
\hline$r$ (mag) & $\ldots$ & $12.269 \pm 0.040$ & $13.490 \pm 0.060$ & $13.579 \pm 0.030$ & APASS $^{d}$ \\
\hline$i$ (mag) & $13.535 \pm 0.010$ & $12.115 \pm 0.040$ & $13.409 \pm 0.070$ & $13.30 \pm 0.28$ & APASS $^{\mathrm{d}}$ \\
\hline$J$ (mag) & $12.643 \pm 0.025$ & $11.241 \pm 0.023$ & $12.523 \pm 0.034$ & $12.458 \pm 0.025$ & 2MASS \\
\hline$H$ (mag) & $12.373 \pm 0.034$ & $10.955 \pm 0.024$ & $12.218 \pm 0.035$ & $12.088 \pm 0.027$ & 2MASS \\
\hline$K_{s}(\mathrm{mag})$ & $12.289 \pm 0.027$ & $10.867 \pm 0.021$ & $12.114 \pm 0.030$ & $12.046 \pm 0.027$ & 2MASS \\
\hline \multicolumn{6}{|l|}{ Derived Properties } \\
\hline$M_{*}\left(M_{*}\right)$ & $1.168 \pm 0.042$ & $1.187 \pm 0.060$ & $1.111 \pm 0.054$ & $0.964 \pm 0.040$ & $\mathrm{YY}+\rho_{*}+\mathrm{ZASPE}^{\mathrm{e}}$ \\
\hline$R_{*}\left(R_{*}\right)$ & $1.117 \pm 0.060$ & $1.44 \pm 0.18$ & $1.046 \pm 0.058$ & $1.101_{-0.024}^{+0.031}$ & $\mathrm{YY}+\rho_{*}+\mathrm{ZASPE}$ \\
\hline $\log g_{*}(\mathrm{cgs})$ & $4.411 \pm 0.038$ & $4.198 \pm 0.088$ & $4.445 \pm 0.034$ & $4.340 \pm 0.019$ & $\mathrm{YY}+\rho_{*}+\mathrm{ZASPE}$ \\
\hline$\rho_{*}\left(\mathrm{~g} \mathrm{~cm}^{-3}\right)^{\mathrm{f}}$ & $1.38_{-0.23}^{+0.16}$ & $0.56_{-0.16}^{+0.21}$ & $1.70_{-0.24}^{+0.17}$ & $1.026_{-0.071}^{+0.048}$ & Light curves \\
\hline$\rho_{*}\left(\mathrm{~g} \mathrm{~cm}^{-3}\right)^{\mathrm{f}}$ & $1.19 \pm 0.16$ & $0.56_{-0.16}^{+0.22}$ & $1.37 \pm 0.18$ & $1.027_{-0.071}^{+0.050}$ & $\begin{array}{l}\text { YY + Light Curves } \\
+ \text { ZASPE }\end{array}$ \\
\hline$L_{*}\left(L_{\odot}\right)$ & $1.39 \pm 0.23$ & $2.04 \pm 0.54$ & $1.17 \pm 0.22$ & $1.11 \pm 0.11$ & $\mathrm{YY}+\rho_{*}+\mathrm{ZASPE}$ \\
\hline$M_{V}(\mathrm{mag})$ & $4.44 \pm 0.19$ & $4.05 \pm 0.27$ & $4.64 \pm 0.22$ & $4.74 \pm 0.12$ & $\mathrm{YY}+\rho_{*}+\mathrm{ZASPE}$ \\
\hline$M_{K}(\mathrm{mag}, \mathrm{ESO})$ & $3.01 \pm 0.13$ & $2.51 \pm 0.26$ & $3.17 \pm 0.15$ & $3.121 \pm 0.066$ & $\mathrm{YY}+\rho_{*}+\mathrm{ZASPE}$ \\
\hline Age (Gyr) & $1.2 \pm 1.1$ & $4.74_{-0.51}^{+0.70}$ & $1.2_{-1.1}^{+1.5}$ & $9.0 \pm 1.9$ & $\mathrm{YY}+\rho_{*}+\mathrm{ZASPE}$ \\
\hline$A_{V}(\mathrm{mag})$ & $0.305 \pm 0.098$ & $0.024_{-0.024}^{+0.059}$ & $0.025_{-0.025}^{+0.108}$ & $0.112 \pm 0.078$ & $\mathrm{YY}+\rho_{*}+\mathrm{ZASPE}$ \\
\hline Distance $(\mathrm{pc})$ & $717 \pm 43$ & $478 \pm 59$ & $631 \pm 44$ & $613 \pm 19$ & $\mathrm{YY}+\rho_{*}+\mathrm{ZASPE}$ \\
\hline
\end{tabular}

Notes. For all four systems, we adopt a model in which the orbit is assumed to be circular. See the discussion in Section 3.3 .

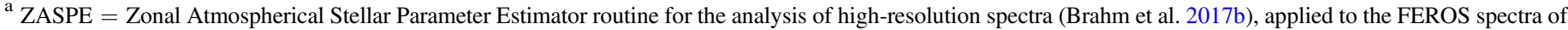

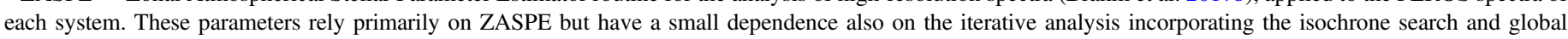
modeling of the data.

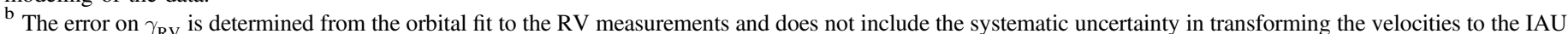
standard system. The velocities have not been corrected for gravitational redshifts.

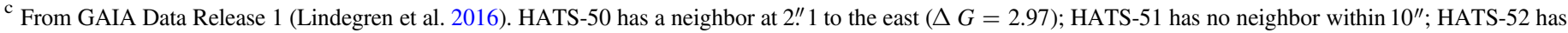
a neighbor at 2 ". 8 to east ( $\Delta G=2.26$ ). HATS-53 has no neighbor within $8^{\prime \prime}$.

${ }^{\mathrm{d}}$ From APASS DR6 for as listed in the UCAC 4 catalog (Zacharias et al. 2012).

e $\mathrm{YY}+\rho_{*}+\mathrm{ZASPE}=$ Based on the YY isochrones (Yi et al. 2001), $\rho_{*}$ as a luminosity indicator, and the ZASPE results

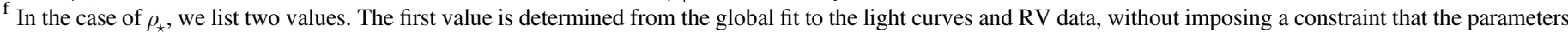

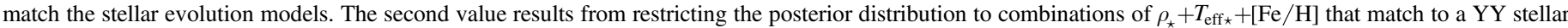
model.

\subsection{Properties of the Parent Star}

As anticipated before, we used high-resolution FEROS spectra for determining the atmospheric properties (metallicity, effective temperature, and surface gravity) of the four stars. The spectra were analyzed with the ZASPE routine, which is comprehensively described in Brahm et al. (2017b).

Then, we followed the methodology of Sozzetti et al. (2007) for determining other stellar parameters (mass, radius, luminosity, age, etc.) together with their uncertainties. In brief, we performed a Markov chain Monte Carlo (MCMC) global analysis of our photometric and spectroscopic data, based on (i) the stellar effective temperature, $T_{\text {eff }}$, and stellar metal abundance, $[\mathrm{Fe} / \mathrm{H}]$, which were both determined with ZASPE, (ii) the stellar mean density, $\rho_{\star}$, estimated by modeling the photometric transit light curves, and (iii) using the Yonsei-Yale (YY; Yi et al. 2001) evolutionary tracks. 
Table 6

Orbital and Planetary Parameters for HATS50b-HATS53b

\begin{tabular}{|c|c|c|c|c|}
\hline Parameter & $\begin{array}{l}\text { HATS-50b } \\
\text { Value }\end{array}$ & $\begin{array}{l}\text { HATS-51b } \\
\text { Value }\end{array}$ & $\begin{array}{l}\text { HATS-52b } \\
\text { Value }\end{array}$ & $\begin{array}{l}\text { HATS-53b } \\
\text { Value }\end{array}$ \\
\hline \multicolumn{5}{|l|}{ Light Curve Parameters } \\
\hline$P$ (days) & $3.8297015 \pm 0.0000046$ & $3.3488702 \pm 0.0000039$ & $1.36665436 \pm 0.00000094$ & $3.8537768 \pm 0.0000038$ \\
\hline$T_{c}(\mathrm{BJD})^{\mathrm{a}}$ & $2456870.34792 \pm 0.00068$ & $2457042.00405 \pm 0.00058$ & $2456929.03039 \pm 0.00033$ & $2457236.75653 \pm 0.00049$ \\
\hline$T_{14}$ (days) $^{\mathrm{a}}$ & $0.1283 \pm 0.0021$ & $0.1384 \pm 0.0020$ & $0.0871 \pm 0.0013$ & $0.1461 \pm 0.0016$ \\
\hline$T_{12}=T_{34}(\text { days })^{\mathrm{a}}$ & $0.0144 \pm 0.0015$ & $0.0138 \pm 0.0017$ & $0.0131 \pm 0.0013$ & $0.01679 \pm 0.00095$ \\
\hline$a / R_{\star}$ & $9.72 \pm 0.44$ & $6.94 \pm 0.74$ & $5.14 \pm 0.22$ & $9.30_{-0.22}^{+0.15}$ \\
\hline$\zeta / R_{\star}^{\mathrm{b}}$ & $17.55 \pm 0.24$ & $16.08 \pm 0.16$ & $26.90 \pm 0.27$ & $15.47 \pm 0.11$ \\
\hline$R_{\mathrm{p}} / R_{\star}$ & $0.1038 \pm 0.0025$ & $0.1010 \pm 0.0038$ & $0.1352 \pm 0.0028$ & $0.1250 \pm 0.0028$ \\
\hline$b^{2}$ & $0.177_{-0.073}^{+0.073}$ & $0.093_{-0.066}^{+0.095}$ & $0.231_{-0.074}^{+0.073}$ & $0.039_{-0.029}^{+0.039}$ \\
\hline$b \equiv a \cos i / R_{\star}$ & $0.421_{-0.099}^{+0.079}$ & $0.30_{-0.14}^{+0.13}$ & $0.481_{-0.084}^{+0.071}$ & $0.198_{-0.096}^{+0.082}$ \\
\hline$i(\mathrm{deg})$ & $87.54 \pm 0.66$ & $87.1 \pm 1.6$ & $84.7 \pm 1.1$ & $88.78 \pm 0.55$ \\
\hline \multicolumn{5}{|l|}{ HATSouth Dilution Factors ${ }^{c}$} \\
\hline Dilution Factor 1 & $0.816 \pm 0.063$ & $0.827 \pm 0.063$ & $0.916 \pm 0.048$ & $0.919 \pm 0.044$ \\
\hline Dilution Factor 2 & $0.880 \pm 0.062$ & $\ldots$ & $\ldots$ & $\ldots$ \\
\hline \multicolumn{5}{|l|}{ Limb-darkening Coefficients ${ }^{\mathrm{d}}$} \\
\hline$c_{1}, r$ & 0.3362 & 0.3801 & 0.3262 & 0.3816 \\
\hline$c_{2}, r$ & 0.3446 & 0.3173 & 0.3487 & 0.3101 \\
\hline 0.3559 & 0.3120 & $\ldots$ & $\ldots$ & 0.3132 \\
\hline$c_{2}, R$ & 0.3470 & $\cdots$ & $\cdots$ & $\cdots$ \\
\hline$c_{1}, i$ & 0.2495 & 0.2833 & 0.2419 & 0.2888 \\
\hline$c_{2}, i$ & 0.3488 & 0.3306 & 0.3502 & 0.3179 \\
\hline \multicolumn{5}{|l|}{ RV Parameters } \\
\hline$K\left(\mathrm{~m} \mathrm{~s}^{-1}\right)$ & $45 \pm 12$ & $94.9 \pm 5.1$ & $380 \pm 23$ & $79 \pm 12$ \\
\hline$e^{\mathrm{e}}$ & $<0.516$ & $<0.330$ & $<0.246$ & $<0.330$ \\
\hline RV Jitter FEROS $\left(\mathrm{m} \mathrm{s}^{-1}\right)^{\mathrm{f}}$ & $69 \pm 11$ & $49 \pm 11$ & $124 \pm 39$ & $18 \pm 12$ \\
\hline RV Jitter HARPS $\left(\mathrm{m} \mathrm{s}^{-1}\right)$ & $\ldots$ & $\ldots$ & $<114.5$ & $<31.0$ \\
\hline RV Jitter CYCLOPS $\left(\mathrm{m} \mathrm{s}^{-1}\right)$ & $\ldots$ & $25.2 \pm 7.8$ & $\ldots$ & $\cdots$ \\
\hline RV Jitter CORALIE $\left(\mathrm{m} \mathrm{s}^{-1}\right)$ & $<286.0$ & $58 \pm 12$ & $\cdots$ & $\cdots$ \\
\hline RV Jitter HIRES $\left(\mathrm{m} \mathrm{s}^{-1}\right)$ & $28 \pm 10$ & $\ldots$ & $\cdots$ & $\ldots$ \\
\hline \multicolumn{5}{|l|}{ Planetary Parameters } \\
\hline$M_{\mathrm{p}}\left(M_{\mathrm{J}}\right)$ & $0.39 \pm 0.10$ & $0.768 \pm 0.045$ & $2.24 \pm 0.15$ & $0.595 \pm 0.089$ \\
\hline$R_{\mathrm{p}}\left(R_{\mathrm{J}}\right)$ & $1.130 \pm 0.075$ & $1.41 \pm 0.19$ & $1.382 \pm 0.086$ & $1.340 \pm 0.056$ \\
\hline$C\left(M_{\mathrm{p}}, R_{\mathrm{p}}\right)^{\mathrm{g}}$ & 0.09 & 0.25 & 0.35 & 0.11 \\
\hline$\rho_{\mathrm{p}}\left(\mathrm{g} \mathrm{cm}^{-3}\right)$ & $0.33 \pm 0.11$ & $0.34_{-0.11}^{+0.16}$ & $1.06 \pm 0.19$ & $0.303 \pm 0.055$ \\
\hline $\log g_{\mathrm{p}}(\mathrm{cgs})$ & $2.87_{-0.14}^{+0.11}$ & $2.98 \pm 0.11$ & $3.468 \pm 0.052$ & $2.912_{-0.087}^{+0.060}$ \\
\hline$a(\mathrm{au})$ & $0.05046 \pm 0.00060$ & $0.04639 \pm 0.00077$ & $0.02498 \pm 0.00040$ & $0.04753 \pm 0.00066$ \\
\hline$T_{\mathrm{eq}}(\mathrm{K})$ & $1348 \pm 47$ & $1553 \pm 92$ & $1834 \pm 73$ & $1312 \pm 25$ \\
\hline$\Theta^{\mathrm{h}}$ & $0.0296 \pm 0.0080$ & $0.0421 \pm 0.0064$ & $0.0725 \pm 0.0064$ & $0.0436_{-0.0073}^{+0.0051}$ \\
\hline $\log _{10}\langle F\rangle(\mathrm{cgs})^{\mathrm{i}}$ & $8.872 \pm 0.060$ & $9.12 \pm 0.10$ & $9.407 \pm 0.069$ & $8.825 \pm 0.033$ \\
\hline
\end{tabular}

Notes. For all four systems, we adopt a model in which the orbit is assumed to be circular. See the discussion in Section 3.3 .

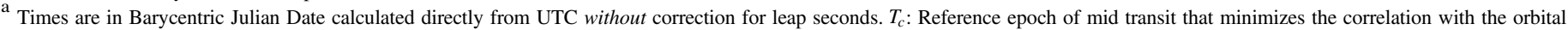
period. $T_{12}$ : total transit duration, time between first to last contact; $T_{12}=T_{34}$ : ingress/egress time, time between first and second, or third and fourth contact.

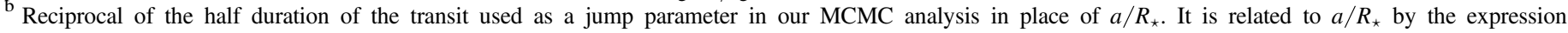
$\zeta / R_{\star}=a / R_{\star}(2 \pi(1+e \sin \omega)) /\left(P \sqrt{1-b^{2}} \sqrt{1-e^{2}}\right)$ (Bakos et al. 2010).

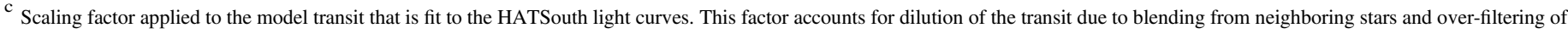

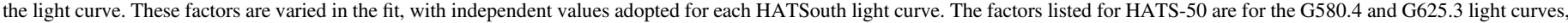
respectively. For HATS-51, we list the factor for 601.2. For HATS-52, the listed factor is for G606.1. For HATS-53, the listed factor is for G610.4.

${ }^{\mathrm{d}}$ Values for a quadratic law, adopted from the tabulations by Claret (2004) according to the spectroscopic (ZASPE) parameters listed in Table 5.

e The 95\% confidence upper limit on the eccentricity determined when $\sqrt{e} \cos \omega$ and $\sqrt{e} \sin \omega$ are allowed to vary in the fit.

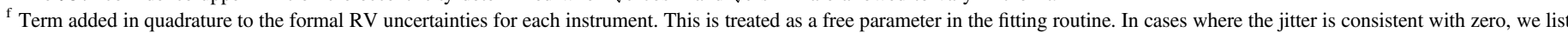
its $95 \%$ confidence upper limit.

${ }^{\mathrm{g}}$ Correlation coefficient between the planetary mass $M_{\mathrm{p}}$ and radius $R_{\mathrm{p}}$ estimated from the posterior parameter distribution.

h The Safronov number is given by $\Theta=\frac{1}{2}\left(V_{\text {esc }} / V_{\text {orb }}\right)^{2}=\left(a / R_{\mathrm{p}}\right)\left(M_{\mathrm{p}} / M_{\star}\right)$ (see Hansen $\&$ Barman 2007).

${ }^{\mathrm{i}}$ Incoming flux per unit surface area, averaged over the orbit.

We determined the YY isochrones for each of the four systems over a wide range of ages. The values of the stellar parameters were obtained from the best agreement between the resulting values of $\rho_{\star}$ and $T_{\text {eff }}$ and those estimated from the data. Figure 10 shows the locations of each star on an $T_{\text {eff } \star}-\rho_{\star}$ diagram. From this analysis, we kept the values of the stellar $\log$ arithmic surface gravities, $\log g_{\star}$, and used them as fixed parameters for a second iteration with ZASPE, which returned the final values of the parameters of the four stars.
They are reported in Table 5, and all of our objects are G-type stars.

The most likely values for $T_{\text {eff } \star}$ and $\rho_{\star}$ for HATS-52 fall at a higher density than the lowest age isochrone tabulated in the models (see bottom left panel in Figure 10). The models and observations are consistent within $2 \sigma$. For determining the physical parameters of this star, we exclude any $T_{\text {eff } \star}-\rho_{\star}-[\mathrm{Fe} / \mathrm{H}]$ point in the Markov chain that does not match to a stellar model. In Table 5, we list for each star the 


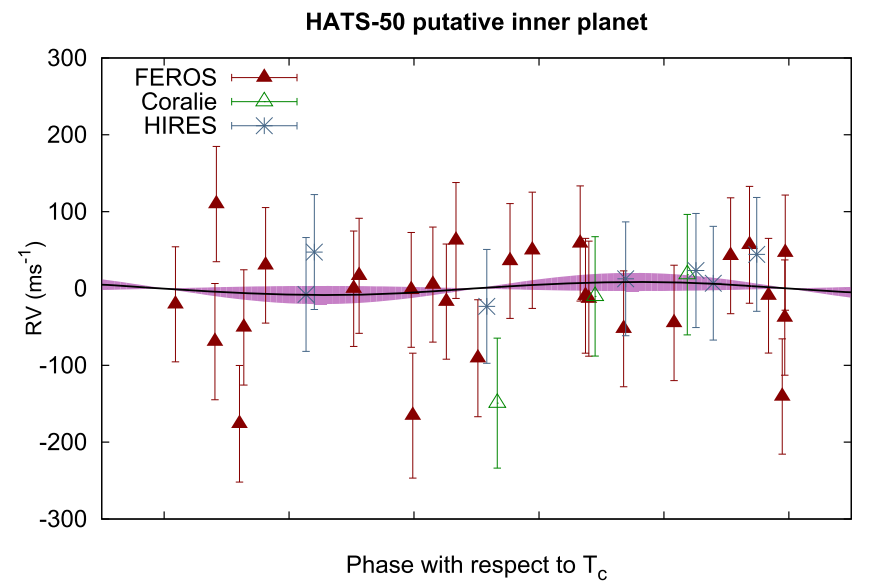

Figure 11. Phased high-precision RV measurements for HATS-50, after subtracting the orbital variation due to the confirmed transiting hot Jupiter, HATS-50b, and phase folded at the period ( 0.77 days) of the candidate inner transiting planet, HATS-50c. The line shows the best-fit circular orbit at this period, while the shaded region shows the $1 \sigma$ uncertainty bounds on this model. The instruments used are labeled in the plots.

median stellar density based both on the full Mark chain (i.e., without enforcing a match to the stellar models) and on the chain after excluding points that do not match to a model.

We note that, while HATS-53 has physical characteristics similar to the Sun $\left(T_{\text {eff } \star}=5644 \pm 94,[\mathrm{Fe} / \mathrm{H}]=0.010 \pm\right.$ $\left.0.066, \quad M_{\star}=0.964 \pm 0.040 M_{\odot}, \quad R_{\star}=1.101_{-0.024}^{+0.031} R_{\odot}\right)$, HATS-50, HATS-51, and HATS-52 are more massive, larger, and metal richer $([\mathrm{Fe} / \mathrm{H}]=0.300 \pm 0.056, \quad[\mathrm{Fe} / \mathrm{H}]=$ $0.300 \pm 0.030$ and $[\mathrm{Fe} / \mathrm{H}]=0.22 \pm 0.10$ for HATS-50, HATS-51, and HATS-52, respectively). We note that HATS-51 is much less dense $\left(\rho_{\star}=0.56_{-0.16}^{+0.22} \mathrm{~g} \mathrm{~cm}^{-3}\right)$ than the other three stars due to its large radius $\left(R_{\star}=1.44 \pm 0.18 R_{\odot}\right)$. Moreover, our analysis indicates that HATS-50 and HATS-52 are quite young, i.e., $1.2 \pm 1.1 \mathrm{Gyr}$ and $1.2_{-1.1}^{+1.5} \mathrm{Gyr}$, respectively; these estimates are both consistent with their Zero Age Main Sequence implying a 95\% confidence upper limit on the age of $t<3.9 \mathrm{Gyr}$ and $t<3.8 \mathrm{Gyr}$, for HATS-50 and HATS-52, respectively. HATS-51 has an intermediate age $\left(4.74_{-0.51}^{+0.70} \mathrm{Gyr}\right)$, whereas HATS-53 is quite old $(9.0 \pm 1.9 \mathrm{Gyr})$.

We also estimated the distance of the four stars by comparing their broad-multi-band photometry taken from public astronomical archives (see Table 5) with the predicted magnitudes in each filter from the isochrones. The extinction was determined assuming an $R_{V}=3.1$ law from Cardelli et al. (1989). For a consistency check, we used the NED online extinction calculator, based on Galactic extinction maps, for estimating the expected total line of site extinction for each source. Three of them (HATS-51, HATS-52, and HATS-53) passed this check, as we found values greater than the inferred $A_{V}$. In the case of HATS-50, our estimated $A_{V}$ is very close to the value determined from the dust maps.

\subsection{Excluding Blend Scenarios}

In order to exclude blend scenarios, we carried out an analysis following Hartman et al. (2012). We attempt to model the available photometric data (including light curves and catalog broad-band photometric measurements) for each object as a blend between an eclipsing binary star system and a third star along the line of sight. The physical properties of the stars are constrained using the Padova isochrones (Girardi et al. 2000), while we also require that the brightest of the three stars in the blend has atmospheric parameters consistent with those measured with ZASPE. We also simulate composite crosscorrelation functions and use them to predict RVs and BSs for each blend scenario considered. The results for each system are as follows:

1. HATS-50 - all blend models tested for this system can be rejected in favor of a model of a single star with a planet with greater than $3 \sigma$ confidence based solely on the photometry. Moreover, the blend models that come closest to fitting the photometric data (those rejected with less than $5 \sigma$ confidence) would yield large BS variations in excess of $1 \mathrm{~km} \mathrm{~s}^{-1}$, whereas the measured scatter in the BS values is only $62 \mathrm{~m} \mathrm{~s}^{-1}$ based on FEROS. Based on this, we conclude that HATS-50 is not a blended stellar eclipsing binary system.

2. HATS-51-we find that the best-fit blend models are indistinguishable from the best-fit planet model based on the photometry. However, all blend models tested that fit the photometry (i.e., those that cannot be rejected in favor of the best-fit single-star plus planet model with at least $5 \sigma$ confidence) would have been easily identified as composite systems based on the spectroscopy, with BS and/or RV variations in excess of $1 \mathrm{~km} \mathrm{~s}^{-1}$. For comparison, the measured FEROS BSs have a scatter of $46 \mathrm{~m} \mathrm{~s}^{-1}$. Based on this, we rule out stellar eclipsing binary blend scenarios.

3. HATS-52-similar to HATS-50, all blend models tested for this system can be rejected in favor of a model of a single star with a planet with greater than $3 \sigma$ confidence based solely on the photometry, while blend models that come closest to fitting the photometric data (those rejected with less than $5 \sigma$ confidence) would yield large BS variations in excess of $1 \mathrm{~km} \mathrm{~s}^{-1}$. In this case, measured scatter in the BS values is $96 \mathrm{~m} \mathrm{~s}^{-1}$ based on FEROS. Based on this, we conclude that HATS-52 is not a blended stellar eclipsing binary system.

4. HATS-53-similar to HATS-50, all blend models tested for this system can be rejected in favor of a model of a single star with a planet with greater than $4 \sigma$ confidence based solely on the photometry, while blend models that come closest to fitting the photometric data (those rejected with less than $5 \sigma$ confidence) would yield large BS variations in excess of $1 \mathrm{~km} \mathrm{~s}^{-1}$. In this case, measured scatter in the BS values is $47 \mathrm{~m} \mathrm{~s}^{-1}$ based on FEROS. Based on this, we conclude that HATS-53 is not a blended stellar eclipsing binary system.

\subsection{Global Modeling of the Data}

The physical parameters of the four planetary systems were estimated by modeling the HATSouth photometry, the follow-up photometry, and the high-precision RV measurements. For this task, we followed the robust procedures developed by the HAT team, which are exhaustively described in several of their exoplanet-discovery papers (e.g., Pál et al. 2008; Bakos et al. 2010; Hartman et al. 2012, 2015). Here, we give a brief summary.

The transit light curves taken by the HATSouth telescopes (Figure 1) were fitted by using Mandel \& Agol (2002) models and considering possible dilution of the transit depth; this was done for taking care of possible (i) blending from neighboring 


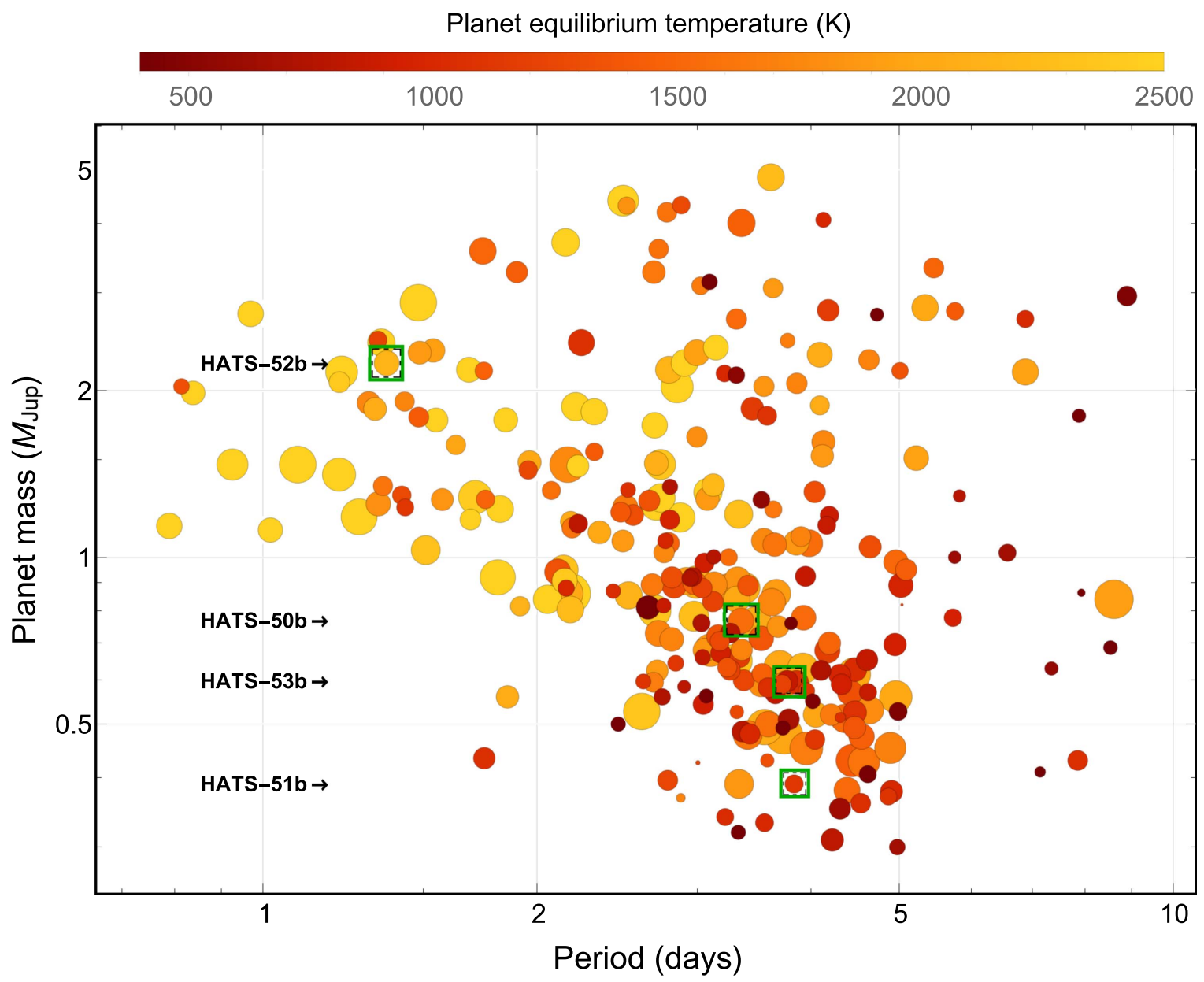

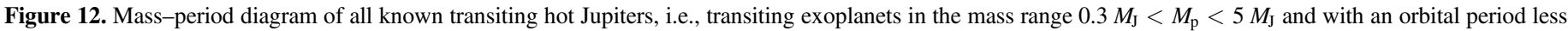

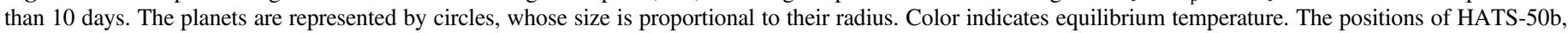

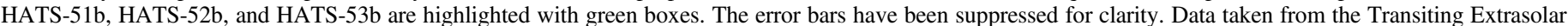
Planet Catalog (TEPCat).

stars, or (ii) over-correction made when the light curves were detrended during the reduction phase.

Concerning the photometric follow-up observations (Figures 4-7), the systematic noise of each data set was corrected during the modeling of the corresponding light curve, by including a quadratic trend in time. We also included linear trends with three parameters describing the measured shape of the PSF. These were included to account for possible variations in the photometry resulting from PSF-shape changes that can happen during the transit observation due to poor guiding or nonphotometric conditions.

The RV curves, which we presented in Section 2.4, are composed of points that were measured with different spectrographs, which can present different zero-points and can be affected by RV jitter as well. Therefore, we modeled the RV curves (Figure 3) with Keplerian orbits considering the zero-point and the RV jitter for each instrument as free parameters.

Finally, the values of the physical parameters of the exoplanetary systems were obtained by exploring their parameter spaces by means of a Differential Evolution MCMC procedure. This allowed us to identify the most likely values for the parameters together with their $1 \sigma$ confidence interval.

We also investigated the possibility that the orbits of the four planets are eccentric. This was done by performing the joint fit of each of the four data sets with both fixed circular orbits and freeeccentricity models. Then, we estimated the Bayesian evidence for each scenario following the method of Weinberg et al. (2013). This method involves using the Markov Chains produced in modeling the observations to identify a region of high posterior probability which dominates the Bayesian evidence, and then carrying out a Monte Carlo integration over this small domain to estimate the evidence. We find that in all cases, a model with a fixed circular orbit has a higher Bayesian evidence than a model where the eccentricity is allowed to vary, and we adopt the fixed circular orbit model for each system.

The resulting parameters for each system are reported in Table 6 and indicate that three of the planets are puffy, lowdensity, hot giants (HATS-50b, HATS-51b, and HATS-53b) with 3 days $<P_{\text {orb }}<4$ days, while the fourth (HATS-52b) is a high-density, massive $\left(M_{\mathrm{p}} \approx 2.2 M_{\mathrm{J}}\right)$, close-in $\left(P_{\text {orb }}=1.37\right.$ days; $a \approx 0.025 \mathrm{au} ; T_{\mathrm{eq}} \approx 1830 \mathrm{~K}$ ) hot Jupiter. The fours planets have a radius larger than Jupiter, and the least massive of the four is HATS-50b with a mass of $\approx 0.4 M_{\mathrm{J}}$.

\subsection{Mass Upper Limit for HATS-50c}

In Section 2.3, we have discussed the possibility that HATS-50 may host another planet (HATS-50c) with a shorter orbital period ( 0.77 days). This putative planet could be the cause of the substantial residuals of the RV measurements of HATS-50, 


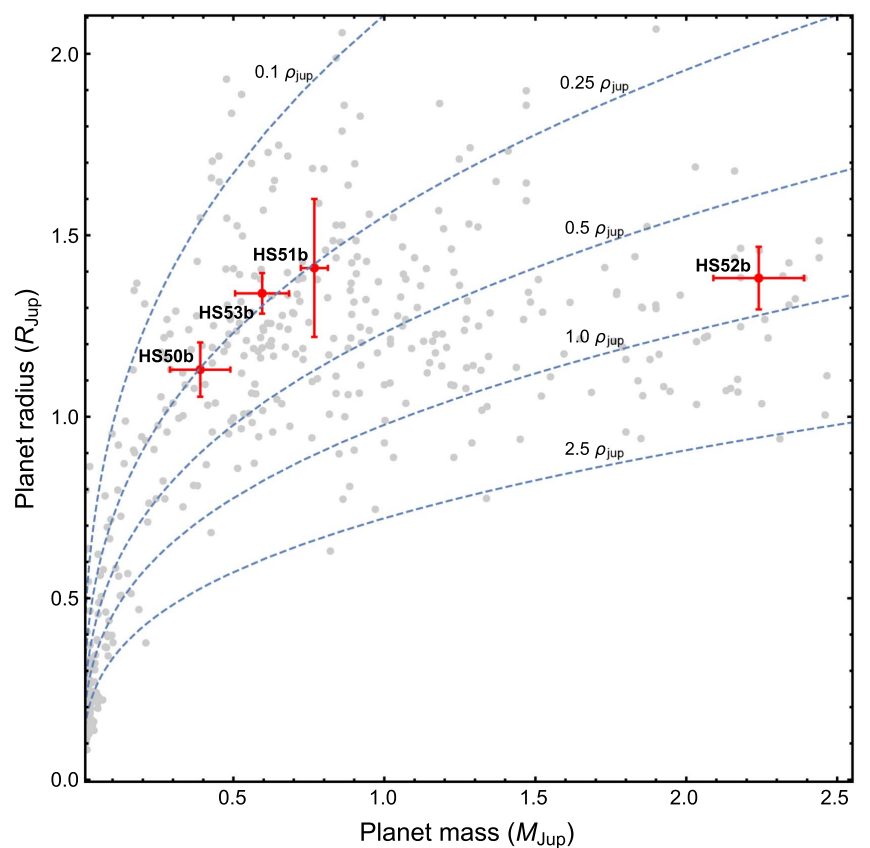

Figure 13. The masses and radii of the known transiting extrasolar planets. The plot is restricted to exoplanets with values of the mass until $2.5 M_{\mathrm{J}}$ and radius until 2.0 $R_{\mathrm{J}}$. Gray points denote values taken from TEPCat. Their error bars have been suppressed for clarity. The new HATS exoplanets, HATS-50b, HATS-51b, HATS-52b, and HATS-53b, are shown in red points with error bars. Dotted lines show where density is $2.5,1.0,0.5,0.25$, and $0.1 \rho_{\mathrm{J}}$.

which are showed in Figure 3 (top left panel) after removing the model for planet $b$. We have therefore deeply investigated this possibility. Figure 11 shows the RVs for HATS-50, after subtracting the orbital variation due to the confirmed transiting hot Jupiter and phase folded at the period of the candidate inner transiting planet. The line shows the best-fit circular orbit at this period, while the shaded region shows the $1 \sigma$ uncertainty bounds on this model. We find that the RVs are consistent with no variation at this period, with a best-fit RV semi-amplitude of $K=8.4 \pm 11.8 \mathrm{~m} \mathrm{~s}^{-1}$. The $95 \%$ confidence upper limit on the mass of the candidate inner transiting planet is thus $M_{\mathrm{pl}, \mathrm{c}}<0.16 M_{\mathrm{J}}$.

\section{Summary and Discussion}

Having now exceeded 50 discoveries, ${ }^{23}$ HATSouth turns out to be one of the most efficient ground-based survey for detecting transiting exoplanets. Thanks to systematic photometric observations of southern-sky regions with the HATSouth robotic telescopes, we have presented the discovery of four new hot Jupiters, namely HATS-50b, HATS-51b, HATS-52b, and HATS-53b. After their detection with the survey facilities, their planetary nature was robustly confirmed through photometric follow-up observations and extensive RV measurements, as described in the previous sections.

All of the photometric and spectroscopic data that we have collected were used for fully characterizing these new exoplanetary systems. From the analysis of the parent stars, we found that they are G-type main-sequence stars and have very different ages. While HATS-50 and HATS-52 are young ( $\approx 1.2$ Gyr), HATS-51 has an age similar to the Sun $(\approx 4.7 \mathrm{Gyr})$, and HATS-51 appears

\footnotetext{
23 The papers describing the discovery of the HATS exoplanets from HATS36 to HATS-49 are under review or close to being submitted.
}

to be very old $(\approx 9.0 \mathrm{Gyr})$. Three of them were found to be metalrich (HATS-50: $[\mathrm{Fe} / \mathrm{H}]=0.300 \pm 0.056 ; \quad$ HATS-51: $[\mathrm{Fe} / \mathrm{H}]=0.300 \pm 0.030 ;$ HATS-52: $[\mathrm{Fe} / \mathrm{H}]=0.22 \pm 0.10$ ), whereas HATS-53 presents a metal abundance similar to the Sun, $[\mathrm{Fe} / \mathrm{H}]=0.010 \pm 0.066$.

Figure 12 shows the positions of the four new HATS planets in the current planet period-mass diagram. They are plotted together with all of the other known transiting hot Jupiters, i.e., exoplanets having a mass in the range $0.3 M_{\mathrm{J}}<M_{\mathrm{p}}<5 M_{\mathrm{J}}$ and an orbital period less than 10 days (data taken from the TEPCat catalog ${ }^{24}$ on 2017 October 30). While HATS-51b and HATS-53b have a similar Safranov number (see Table 6) and are located in regions of the diagram where the hot Jupiters are very packed, HATS-50b and HATS-52b are in less-populated regions of the diagram, highlighting the well-known desert of low-mass Jupiters and Neptunes at low orbital periods (e.g., Mazeh et al. 2005; BenítezLlambay et al. 2011; Mazeh et al. 2016).

The inflated size of HATS-50b, HATS-51b, and HATS-53b is evident from Figure 13, in which the mass-radius diagram of known transiting exoplanets (with mass and radius up to $2.5 M_{\mathrm{J}}$ and $2.0 R_{\mathrm{J}}$, respectively) is shown. The three planets exhibit a similar density. Instead, due to its mass, HATS-52b occupies a zone a slightly apart from the other three and from the crowd of giant exoplanets, similar to the physical characteristics of WASP-36b (Mancini et al. 2016) and Kepler-17b (Désert et al. 2011). Moreover, as the stellar radiation that it receives from its star is $\approx 2.6 \times 10^{9} \mathrm{erg} \mathrm{s}^{-1}$, HATS-52b is very hot $\left(T_{\mathrm{eq}}=1834 \pm 73 \mathrm{~K}\right)$ and belongs to the pM class of hot Jupiters, according to the terminology of Fortney et al. $(2008) .^{25}$

The panels of Figure 14 show the position of the four planets in the mass-density diagram of the currently known transiting exoplanets. Each planet is compared with five different theoretical models estimated by Fortney et al. (2007). Each model has a different core of heavy-elements, i.e., 0, 10, 25, 50, and 100 Earth mass and each panel shows models that were estimated for different values of planet-star separation and stellar age, as explained in the caption of the figure. The four planets have densities comparable with models of core-free planets. One potential explanation could be that the planets are bloated, which would provide the incorrect impression of a core mass that is too small (Thorngren \& Fortney 2017). An alternative explanation would be that relatively low opacities would allow gas runaway accretion also for lower core masses (Mordasini 2014; Ormel 2014). In a recent investigation based on results of the Juno mission, the core mass of Jupiter was estimated to be in the range between 7 and 25 Earth mass (Wahl et al. 2017), which points to a relatively small core mass.

Finally, we would like to remark the possible existence of an inner planet in the HATS-50 planetary system. The analysis of the photometric data of the HATSouth survey has actually revealed a small transit signal with duration of 46 minutes (see Figure 2), yet with an SDE below our threshold for selecting it as a planet candidate. The radius, estimated from the bestfitting model of the HATS photometry and the upper limit of its mass, as coming from the RV measurements, suggest that this

\footnotetext{
${ }^{24}$ The Transiting Extrasolar Planet Catalog (TEPCat) is available at http:// www.astro.keele.ac.uk/jkt/tepcat/ (Southworth 2011).

25 The hypothesis proposed by Fortney et al. (2008) is to divide hot Jupiters into two classes (pM- and pL-class planets, analogous to the M- and L-type dwarfs), depending on the presence, in their atmospheres, of strong absorbers such as gaseous $\mathrm{TiO}$ and $\mathrm{VO}$.
} 

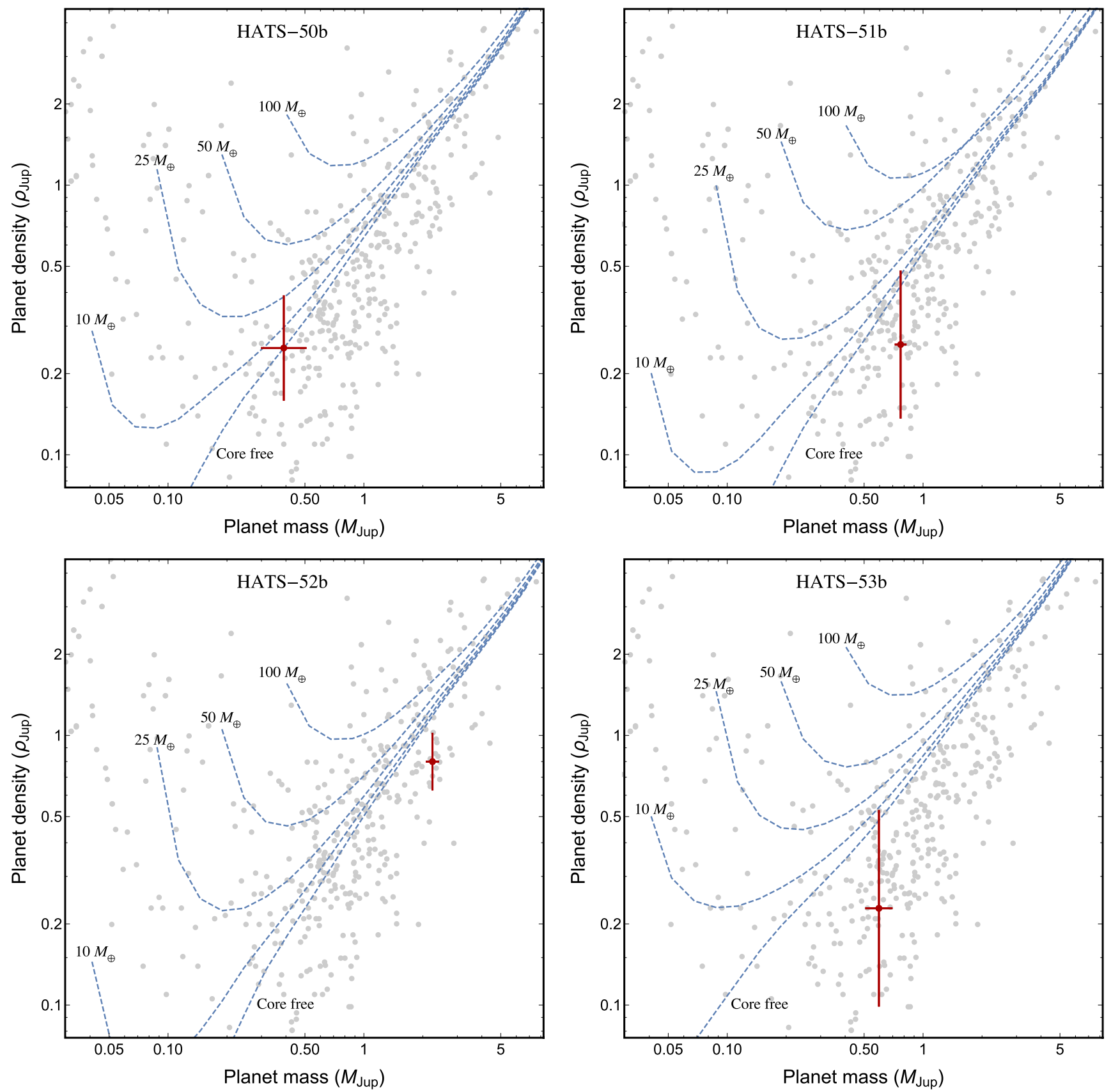

Figure 14. The mass-density diagram of the currently known transiting exoplanets. The gray points denote values taken from TEPCat. Their error bars have been suppressed for clarity. The position of HATS-50b, HATS-51b, HATS-52b, and HATS-53b are shown in red with error bars in the top left, top right, bottom left, and bottom right panels, respectively. Four planetary models, with various heavy-element core masses (10, 25, 50, and 100 Earth mass) and another without a core (Fortney et al. 2007), are plotted for comparison. They were estimated for a planet at 0.045 au from a parent star with an age of $1.0 \mathrm{Gyr}$ (top left panel), 0.045 au and $3.16 \mathrm{Gyr}$ (top right panel), 0.02 au and $1.0 \mathrm{Gyr}$ (bottom left panel), and $0.045 \mathrm{au}$ and $10 \mathrm{Gyr}$ (bottom right panel).

putative planet $\mathrm{c}$ has physical characteristics of a super Neptune. Its short periodicity ( 0.77 days $)$ places it in the Neptune desert, thus making it an interesting candidate to possibly confirm or invalidate with more performing astronomical facilities, as the next space telescope TESS will be.

Development of the HATSouth project was funded by NSF MRI grant NSF/AST-0723074, operations have been supported by NASA grants NNX09AB29G, NNX12AH91H, and NNX17AB61G, and follow-up observations receive partial support from grant NSF/AST-1108686. J.H. acknowledges support from NASA grant NNX14AE87G. A.J. acknowledges support from FONDECYT project 1171208, BASAL CATA PFB-06, and project IC120009 "Millennium Institute of Astrophysics (MAS)" of the Millennium Science Initiative, Chilean Ministry of Economy. N.E. is supported by CONICYT-PCHA/ Doctorado Nacional. R.B. and N.E. acknowledge support from project IC120009 "Millennium Institute of Astrophysics (MAS)" of the Millennium Science Initiative, Chilean Ministry of Economy. V.S. acknowledges support form BASAL CATA PFB-06. A.V. is supported by the NSF Graduate Research Fellowship, grant No. DGE 1144152. This work also uses observations obtained with facilities of the Las Cumbres Observatory Global Telescope (LCOGT). This work is based on 
observations collected with HARPS at the European Organisation for Astronomical Research in the Southern Hemisphere under ESO programme 095.C-0367. This work has made use of data from the European Space Agency (ESA) mission Gaia (https:// www.cosmos.esa.int/gaia), processed by the Gaia Data Processing and Analysis Consortium (DPAC, https://www.cosmos.esa. int/web/gaia/dpac/consortium). Funding for the DPAC has been provided by national institutions, in particular the institutions participating in the Gaia Multilateral Agreement. We acknowledge the use of the AAVSO Photometric All-Sky Survey (APASS), funded by the Robert Martin Ayers Sciences Fund, and the SIMBAD database, operated at CDS, Strasbourg, France. Operations at the MPG $2.2 \mathrm{~m}$ Telescope are jointly performed by the Max Planck Gesellschaft and the European Southern Observatory in La Silla. We thank the MPG $2.2 \mathrm{~m}$ telescope support team for their technical assistance during observations." This work is based in part on observations carried out with the Keck I telescope at Mauna Kea Observatory in Hawaii. Time on this facility was awarded through the Australian community access. Australian community access to the Keck Observatory was supported through the Australian Government's National Collaborative Research Infrastructure Strategy, via the Department of Education and Training, and an Australian Government astronomy research infrastructure grant, via the Department of Industry and Science. The authors wish to thank the anonymous referee for his or her useful comments.

\section{ORCID iDs}

L. Mancini (ib https://orcid.org/0000-0002-9428-8732 G. Á. Bakos (iD https://orcid.org/0000-0001-7204-6727 J. D. Hartman (1D https://orcid.org/0000-0001-8732-6166 W. Bhatti (i) https://orcid.org/0000-0002-0628-0088 R. Brahm (i) https://orcid.org/0000-0002-9158-7315 M. de Val-Borro (i) https://orcid.org/0000-0002-0455-9384

N. Espinoza (1) https://orcid.org/0000-0001-9513-1449

B. J. Fulton (1D https://orcid.org/0000-0003-3504-5316

A. W. Howard (iD https://orcid.org/0000-0001-8638-0320

A. Jordán (i) https://orcid.org/0000-0002-5389-3944

G. W. Marcy (1) https://orcid.org/0000-0002-2909-0113

K. Penev (iD https://orcid.org/0000-0003-4464-1371

M. Rabus (Di) https:// orcid.org/0000-0003-2935-7196

V. Suc (1) https://orcid.org/0000-0001-7070-3842

C. G. Tinney (I) https://orcid.org/0000-0002-7595-0970

S. Durkan (i) https://orcid.org/0000-0002-3663-3251

\section{References}

Addison, B. C., Tinney, C. G., Wright, D. J., et al. 2013, ApJL, 774, L9 Alsubai, K. A., Parley, N. R., Bramich, D. M., et al. 2013, AcA, 63, 465 Bakos, G. Á, Csubry, Z., Penev, K., et al. 2013, PASP, 125, 154 Bakos, G. Á, Noyes, R. W., Kovács, G., et al. 2004, PASP, 116, 266 Bakos, G. Á, Penev, K., Bayliss, D., et al. 2015, ApJ, 813, 111 Bakos, G. Á, Torres, G., Pál, A., et al. 2010, ApJ, 710, 1724 Batygin, K., Bodenheimer, P. H., \& Laughlin, G. P. 2016, ApJ, 829, 114 Bayliss, D., Hartman, J. D., Bakos, G. Á, et al. 2015, AJ, 150, 49 Bayliss, D., Zhou, G., Penev, K., et al. 2013, AJ, 146, 113 Becker, J. C., Vanderburg, A., Adams, F. C., Rappaport, S. A., \& Schwengeler, H. M. 2015, ApJL, 812, L18

Benítez-Llambay, P., Masset, F., \& Beaugé, C. 2011, A\&A, 528, A2

Berta-Thompson, Z. K., Irwin, J., Charbonneau, D., et al. 2015, Natur, 527, 204 Bitsch, B., \& Kley, W. 2011, A\&A, 530, A41

Bodenheimer, P., Hubickyj, O., \& Lissauer, J. J. 2000, Icar, 143, 2 Boley, A. C., Granados Contreras, A. P., \& Gladman, B. 2016, ApJL, 817, L17 Borucki, W. J., Koch, D. G., Basri, G., et al. 2011, ApJ, 736, 19
Brahm, R., Jordán, A., Bakos, G. Á, et al. 2016, AJ, 151, 89 Brahm, R., Jordán, A., \& Espinoza, N. 2017a, PASP, 129, 034002 Brahm, R., Jordán, A., Hartman, J., \& Bakos, G. 2017b, MNRAS, 467, 971 Brown, T. M., Baliber, N., Bianco, F. B., et al. 2013, PASP, 125, 1031 Burke, C. J., McCullough, P. R., Valenti, J. A., et al. 2007, ApJ, 671, 2115 Cardelli, J. A., Clayton, G. C., \& Mathis, J. S. 1989, ApJ, 345, 245 Charbonneau, D., Berta, Z. K., Irwin, J., et al. 2009, Natur, 462, 891 Chatterjee, S., Ford, E. B., Matsumura, S., \& Rasio, F. A. 2008, ApJ, 686, 580

Claret, A. 2004, A\&A, 428, 1001

Damasso, M., Biazzo, K., Bonomo, A. S., et al. 2015, A\&A, 575, A111 Désert, J.-M., Charbonneau, D., Demory, B.-O., et al. 2011, ApJS, 197, 14 Desidera, S., Bonomo, A. S., Claudi, R. U., et al. 2014, A\&A, 567, L6 Dopita, M., Hart, J., McGregor, P., et al. 2007, Ap\&SS, 310, 255 Dressing, C. D., \& Charbonneau, D. 2013, ApJ, 767, 95

Espinoza, N., Bayliss, D., Hartman, J. D., et al. 2016, AJ, 152, 108 Fabrycky, D., \& Tremaine, S. 2007, ApJ, 669, 1298

Fortney, J. J., Lodders, K., \& Marley, M. S. 2008, ApJ, 678, 1419 Fortney, J. J., Marley, M. S., \& Barnes, J. W. 2007, ApJ, 659, 1661 Fressin, F., Torres, G., Charbonneau, D., et al. 2013, ApJ, 766, 81 Gaudi, B. S., Stassun, K. G., Collins, K. A., et al. 2017, Natur, 546, 514 Gillon, M., Jehin, E., Lederer, S. M., et al. 2016, Natur, 533, 221

Girardi, L., Bressan, A., Bertelli, G., \& Chiosi, C. 2000, A\&AS, 141, 371 Hansen, B. M. S., \& Barman, T. 2007, ApJ, 671, 861

Hartman, J. D., Bayliss, D., Brahm, R., et al. 2015, AJ, 149, 166 Hartman, J. D., Bakos, G. Á, Béky, B., et al. 2012, AJ, 144, 139 Hebb, L., Collier-Cameron, A., Loeillet, B., et al. 2009, ApJ, 693, 1920

Hellier, C., Anderson, D. R., Collier Cameron, A., et al. 2012, MNRAS, 426, 739

Henden, A. A., Welch, D. L., Terrell, D., \& Levine, S. E. 2009, in AAS Meeting 214 Abstracts, 407.02

Hippler, S., Bergfors, C., Brandner, W., et al. 2009, Msngr, 137, 14 Howard, A. W., Johnson, J. A., Marcy, G. W., et al. 2010, ApJ, 721, 1467 Janson, M., Durkan, S., Hippler, S., et al. 2017, A\&A, 599, A70 Jordán, A., Brahm, R., Bakos, G. Á, et al. 2014, AJ, 148, 29 Kaufer, A., \& Pasquini, L. 1998, Proc. SPIE, 3355, 844

Kovács, G., Bakos, G., \& Noyes, R. W. 2005, MNRAS, 356, 557

Kovács, G., Zucker, S., \& Mazeh, T. 2002, A\&A, 391, 369

Lin, D. N. C., Bodenheimer, P., \& Richardson, D. C. 1996, Natur, 380, 606

Lindegren, L., Lammers, U., Bastian, U., et al. 2016, A\&A, 595, A4 Mancini, L., Kemmer, J., Southworth, J., et al. 2016, MNRAS, 459, 1393 Mandel, K., \& Agol, E. 2002, ApJL, 580, L171

Marzari, F., \& Nelson, A. F. 2009, ApJ, 705, 1575

Mayor, M., Pepe, F., Queloz, D., et al. 2003, Msngr, 114, 20

Mazeh, T., Holczer, T., \& Faigler, S. 2016, A\&A, 589, A75

Mazeh, T., Zucker, S., \& Pont, F. 2005, MNRAS, 356, 955

Mohler-Fischer, M., Mancini, L., Hartman, J. D., et al. 2003, A\&A, 558, A55 Mordasini, C. 2014, A\&A, 572, A118

Ormel, C. W. 2014, ApJL, 789, L18

Pál, A., Bakos, G. Á, Torres, G., et al. 2008, ApJ, 680, 1450 Penev, K., Bakos, G. Á, Bayliss, D., et al. 2013, AJ, 145, 5

Penev, K., Hartman, J. D., Bakos, G. Á, et al. 2016, AJ, 152, 127

Pepper, J., Kuhn, R. B., Siverd, R., et al. 2012, PASP, 124, 230

Pepper, J., Pogge, R. W., DePoy, D. L., et al. 2007, PASP, 119, 923

Pollacco, D. L., Skillen, I., Collier Cameron, A., et al. 2006, PASP, 118, 1407 Queloz, D., Mayor, M., Udry, S., et al. 2001, Msngr, 105, 1

Rasio, F. A., \& Ford, E. B. 1996, Sci, 274, 954

Ricker, G. R. \& TESS Science Team 2017, in AAS Meeting 229 Abstracts, 104.09

Sedaghati, E., Boffin, H. M. J., MacDonald, R. J., et al. 2017, Natur, 549, 238 Sing, D. K., Fortney, J. J., Nikolov, N., et al. 2016, Natur, 529, 59 Southworth, J. 2011, MNRAS, 417, 2166

Sozzetti, A., Torres, G., Charbonneau, D., et al. 2007, ApJ, 664, 1190 Thorngren, D. P., \& Fortney, J. J. 2017, AJ, submitted (arXiv:1709.04539) Vogt, S. S., Allen, S. L., Bigelow, B. C., et al. 1994, Proc. SPIE, 2198, 362 Wahl, S. M., Hubbard, W. B., Militzer, B., et al. 2017, GeoRL, 44, 4649 Weinberg, M. D., Yoon, I., \& Katz, N. 2013 (arXiv:1301.3156)

Wheatley, P. J., West, R. G., Goad, M. R., et al. 2017, MNRAS, in press (arXiv: 1710.11100)

Yi, S., Demarque, P., Kim, Y.-C., et al. 2001, ApJS, 136, 417

Zacharias, N., Finch, C. T., Girard, T. M., et al. 2012, yCat, 1322, 0

Zechmeister, M., \& Kürster, M. 2009, A\&A, 496, 577

Zhou, G., Bayliss, D., Hartman, J. D., et al. 2014, MNRAS, 437, 2831

Zhou, G., Bayliss, D., Hartman, J. D., et al. 2015, MNRAS, 451, 2263 